\title{
Heterogeneously-Grown Tunable Tensile Strained Germanium on Silicon for Photonic Devices
}

\author{
Michael Clavel, ${ }^{\dagger}$ Dzianis Saladukha, ${ }^{\ddagger}$, Patrick S. Goley, $^{\dagger}$ Tomasz J. Ochalski, ${ }^{\ddagger} \S$ Felipe Murphy-Armando, \\ Robert J. Bodnar, "and Mantu K. Hudait* ${ }^{\dagger}$ \\ ${ }^{\dagger}$ Advanced Devices \& Sustainable Energy Laboratory (ADSEL), Bradley Department of Electrical and Computer Engineering, \\ Virginia Tech, Blacksburg, Virginia 24061, United States \\ ${ }^{\ddagger}$ Tyndall National Institute, Lee Maltings, Dyke Parade, Cork, Ireland \\ ${ }^{\S}$ Centre for Advanced Photonics and Process Analysis, Cork Institute of Technology, Cork, Ireland \\ "Fluids Research Laboratory, Department of Geosciences, Virginia Tech, Blacksburg, Virginia 24061, United States
}

ABSTRACT: The growth, structural and optical properties, and energy band alignments of tensile-strained germanium ( $\varepsilon$-Ge) epilayers heterogeneously integrated on silicon $(\mathrm{Si})$ were demonstrated for the first time. The tunable $\varepsilon$-Ge thin films were achieved using a composite linearly graded $\operatorname{In}_{x} \mathrm{Ga}_{1-x} \mathrm{As} / \mathrm{GaAs}$ buffer architecture grown via solid source molecular beam epitaxy. Highresolution X-ray diffraction and micro-Raman spectroscopic analysis confirmed a pseudomorphic $\varepsilon$-Ge epitaxy whereby the degree of strain varied as a function of the $\mathrm{In}_{x} \mathrm{Ga}_{1-x}$ As buffer indium alloy composition. Sharp heterointerfaces between each $\varepsilon$-Ge epilayer and the respective $\operatorname{In}_{x} \mathrm{Ga}_{1-x}$ As strain template were confirmed by detailed strain analysis using cross-sectional transmission electron microscopy. Low-temperature microphotoluminescence measurements confirmed both direct and indirect bandgap radiative recombination between the $\Gamma$ and $\mathrm{L}$ valleys of $\mathrm{Ge}$ to the light-hole valence band, with $\mathrm{L}-\mathrm{lh}$ bandgaps of 0.68 and $0.65 \mathrm{eV}$

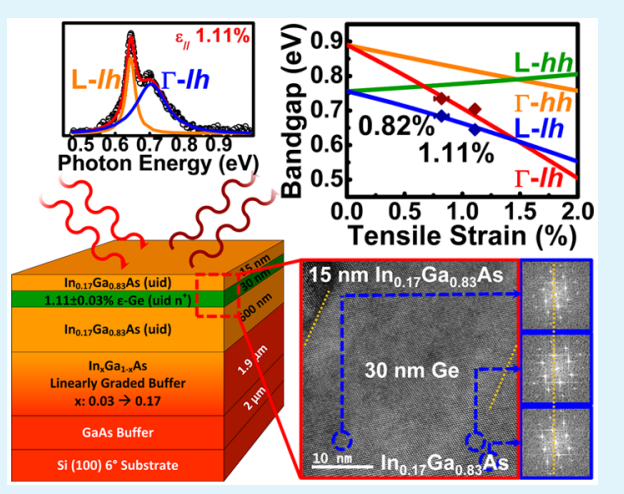
demonstrated for the $0.82 \pm 0.06 \%$ and $1.11 \pm 0.03 \%$ strained $\mathrm{Ge}$ on $\mathrm{Si}$, respectively. Type-I band alignments and valence band offsets of 0.27 and $0.29 \mathrm{eV}$ for the $\varepsilon$ - $\mathrm{Ge} / \mathrm{In}_{0.11} \mathrm{Ga}_{0.89} \mathrm{As}(0.82 \%)$ and $\varepsilon$ - $\mathrm{Ge} / \mathrm{In}_{0.17} \mathrm{Ga}_{0.83} \mathrm{As}(1.11 \%)$ heterointerfaces, respectively, show promise for $\varepsilon$-Ge carrier confinement in future nanoscale optoelectronic devices. Therefore, the successful heterogeneous integration of tunable tensile-strained $\mathrm{Ge}$ on $\mathrm{Si}$ paves the way for the design and implementation of novel Ge-based photonic devices on the Si technology platform.

KEYWORDS: germanium, optical domain, efficient cost-effective light sources, laser integration, molecular beam epitaxy, heterostructure

\section{INTRODUCTION}

The shrinking feature size of silicon $(\mathrm{Si})$ transistors has enabled an exponential increase in transistor density, resulting in increased compute power. However, current Si-based complementary metal-oxide-semiconductor (CMOS) technology is nearing the physical limits of its scaling potential, and with the end in sight of the traditional technology roadmap, ${ }^{1}$ only a radical departure from Si-based technologies can ensure continued technological progress. ${ }^{2} \mathrm{New}$ material innovations, ${ }^{3-5}$ novel device architectures, ${ }^{6-9}$ heterogeneous technology cointegration, ${ }^{10}$ new functionalities, ${ }^{11}$ and their monolithic integration onto $\mathrm{Si}$ are projected to continue transistor miniaturization beyond the $\mathrm{Si}$ CMOS era. Moreover, interconnect bottlenecks for both interchip and intrachip communication are projected to be major impediments to energy-efficient performance scaling. Therefore, there is an urgent need for large bandwidth, low-resistance interconnects in high-end computing applications as copper-based electrical interconnects are rapidly becoming inefficient in meeting essential bandwidth requirements. ${ }^{12}$ It will become increasingly challenging to transmit signals electrically while maintaining low power consumption, low delay, and a high signal-to-noise ratio. ${ }^{13}$ An enticing approach would be the integration of photonic devices with $\mathrm{Si}$ technology, hence the monolithic integration of Si-based optoelectronics would be an obvious choice. However, the indirect bandgap of $\mathrm{Si}$ limits the realization of Si-based photonic devices. ${ }^{14}$ Thus, the hybrid integration of germanium (Ge) and III-V materials-based optoelectronic devices with traditional Si CMOS technology would revolutionize technology needs in the near future. The superior transport properties and large modulation bandwidth of $\mathrm{Ge}$ and III-V compound semiconductor material systems make them ideal candidates for integration on $\mathrm{Si}^{15-21}$ Besides, Ge-based light sources on Si are important for the realization of future, nanoscale optical on-chip communication. ${ }^{22-24}$ Furthermore, optical interconnects compatible with $\mathrm{Si}$ process technology are needed to provide for ever-increasing future bandwidth needs. ${ }^{25}$ Moreover, the electrical-to-optical inter-

Received: August 10, 2015

Accepted: November 12, 2015 

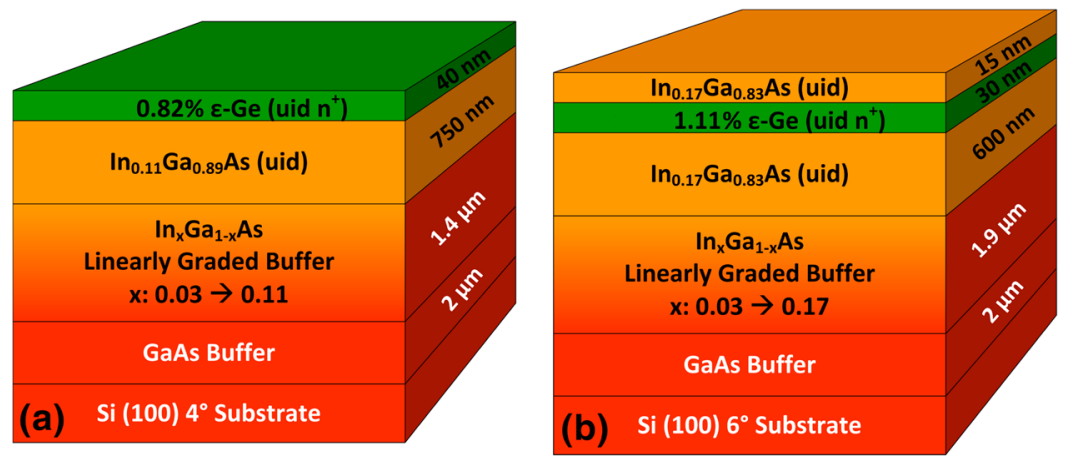

Figure 1. Cross-sectional schematic of (a) $0.82 \% \varepsilon$-Ge/ $\mathrm{In}_{0.11} \mathrm{Ga}_{0.89} \mathrm{As}$ and (b) $1.11 \% \varepsilon$-Ge/ $\mathrm{In}_{0.17} \mathrm{Ga}_{0.83} \mathrm{As}$ structures heterogeneously integrated onto Si.

connect transition for chip-to-chip communication is expected to be a gradual process depending upon specific application requirements and cost-performance trade-offs with current copper-based interconnect technologies. ${ }^{26}$

Currently, the III-V laser bonded to $\mathrm{Si}$ waveguide approach $^{27,28}$ imposes strict design and process constraints, with limited scalability of size (because of the evanescently coupled lasing mode) and cost (because of III-V wafer area utilization in large channel count transceivers). A heterogeneously grown active material with the scope for a tunable wavelength laser would alleviate these constraints and provide a more scalable solution as device size decreases and integration density increases. Very recently, lasing from tensile strained direct-bandgap $\mathrm{Ge}_{1-x} \mathrm{Sn}_{x}$ alloys on $\mathrm{Si}^{29}$ and its potential applications in optoelectronics as well as transistors ${ }^{30,31}$ has gained widespread research interest due to the modification of the $\mathrm{Ge}_{1-x} \mathrm{Sn}_{x}$ bandgap both by tin $(\mathrm{Sn})$ incorporation and strain modulation. The direct bandgap $\mathrm{Ge}_{1-x} \mathrm{Sn}_{x}(x>0.1)$ material can be used as a gain medium in tunable wavelength lasers due to the modulation of the direct $\Gamma$ valley bandgap for increasing Sn compositions; however, the thermal stability of $\mathrm{Ge}_{1-x} \mathrm{Sn}_{x}$ because of $\mathrm{Sn}$ segregation ${ }^{31}$ as well as the lower $\mathrm{Ge}_{1-x} \mathrm{Sn}_{x}$ growth temperature imposes great difficulties for process integration with state-of-the-art $\mathrm{Si}$ device fabrication processes. Therefore, there is a critical need to develop a material system that will be compatible with industry-standard Si process flows, including higher process temperature stability, and provides a feasible template for strain-engineered bandgap modulation for on-Si light sources. Furthermore, light sources with directly grown active materials are needed for the largescale integration of highly integrated photonic devices for optical interconnects in future high-capacity datacenters, warehouse-scale computing, and high-performance computing applications. In this paper, we have demonstrated a heterogeneous integration scheme for tunable tensile-strained $\mathrm{Ge}(\varepsilon-\mathrm{Ge})$ on $\mathrm{Si}$ using variable surface-terminated lattice constant buffering of III-V compound semiconductor metamorphic layers that shows great promise for realizing modular group-IV based lasers on Si. In our hybrid Ge and III$\mathrm{V}$ on $\mathrm{Si}$ integration approach, we have simultaneously combined a substrate architecture that offers a greater design flexibility with diverse material choices to produce scalable, heterogeneously integrated tunable light sources on $\mathrm{Si}$ that target the possibility of realizing hybrid electronic and photonic systems on $\mathrm{Si}$ for ultralow power computing, interchip communication, and optoelectronic applications. We are able to achieve these attributes by carefully controlling the modular tensile strain in the Ge epitaxial layers during growth, and in tandem with the strain analysis, defect examination, morphological, and band alignment properties of these strained Ge material systems, provide a path to achieve group-IV-based lasers on Si.

\section{RESULTS AND DISCUSSION}

Strain Analysis via XRD. Figures $1 \mathrm{a}$ and $1 \mathrm{~b}$ show the schematic diagrams of the $\varepsilon$-Ge/ $/ \operatorname{In}_{x} \mathrm{Ga}_{1-x}$ As heterostructures that were heterogeneously grown on off-cut (100) Si substrates and studied in this work. For the $1.11 \% \varepsilon$-Ge sample, a $15 \mathrm{~nm}$ constant composition $\mathrm{In}_{0.17} \mathrm{Ga}_{0.83}$ As capping layer was grown in order to study the materials and optical properties of $\mathrm{In}_{0.17} \mathrm{Ga}_{0.83}$ As cladded $\varepsilon$-Ge optical cavities. The strain relaxation properties of these structures were investigated using high-resolution X-ray diffractometry (HR-XRD). Figure 2

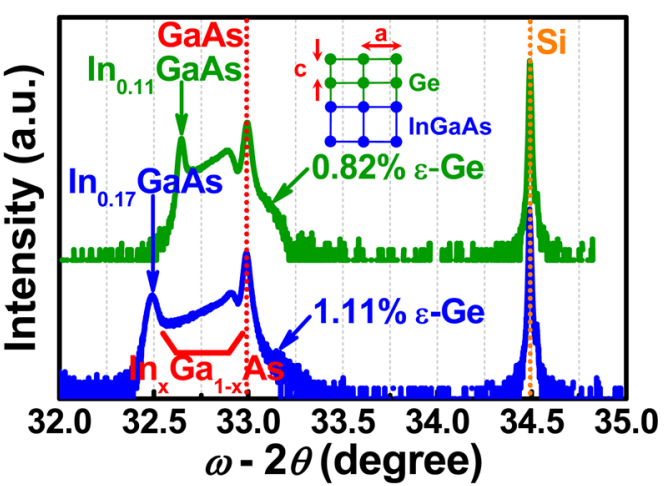

Figure 2. X-ray rocking curves from the $0.82 \%$ (green) and $1.11 \%$ (blue) $\varepsilon$-Ge on Si structures showing movement of the Ge Bragg angle with increasing tensile strain. Inset demonstrates the effect of heteroepitaxial mismatch on the in-plane lattice constant of the toplying epilayer.

shows the high-resolution triple axis symmetric (004) X-ray rocking curves (RCs) from the 30 to $40 \mathrm{~nm} \varepsilon$-Ge epilayers grown on (i) $750 \mathrm{~nm} \mathrm{In}_{0.11} \mathrm{Ga}_{0.89} \mathrm{As} / 1.4 \mu \mathrm{m}$ graded $\mathrm{In}_{x} \mathrm{Ga}_{1-x} \mathrm{As} /$ $2 \mu \mathrm{m} \mathrm{GaAs}$ (0.82\% tensile strain, green) and (ii) $600 \mathrm{~nm}$ $\mathrm{In}_{0.17} \mathrm{Ga}_{0.83} \mathrm{As} / 1.9 \mu \mathrm{m}$ graded $\mathrm{In}_{x} \mathrm{Ga}_{1-x} \mathrm{As} / 2 \mu \mathrm{m}$ GaAs $(1.11 \%$ tensile strain, blue) metamorphic buffers on $\mathrm{Si}$, respectively. Additionally, each diffraction peak in Figure 2 is labeled with its corresponding epilayer. Likewise, Figure 3 shows the symmetric (004) and asymmetric (115) RSMs of the $\varepsilon$-Ge/ $\mathrm{In}_{0.11} \mathrm{Ga}_{0.89} \mathrm{As}$ on Si structure, while Figure 4 similarly shows the (004) and (115) RSMs taken from the $\varepsilon$ - $\mathrm{Ge} / \mathrm{In}_{0.17} \mathrm{Ga}_{0.83}$ As on Si structure. 


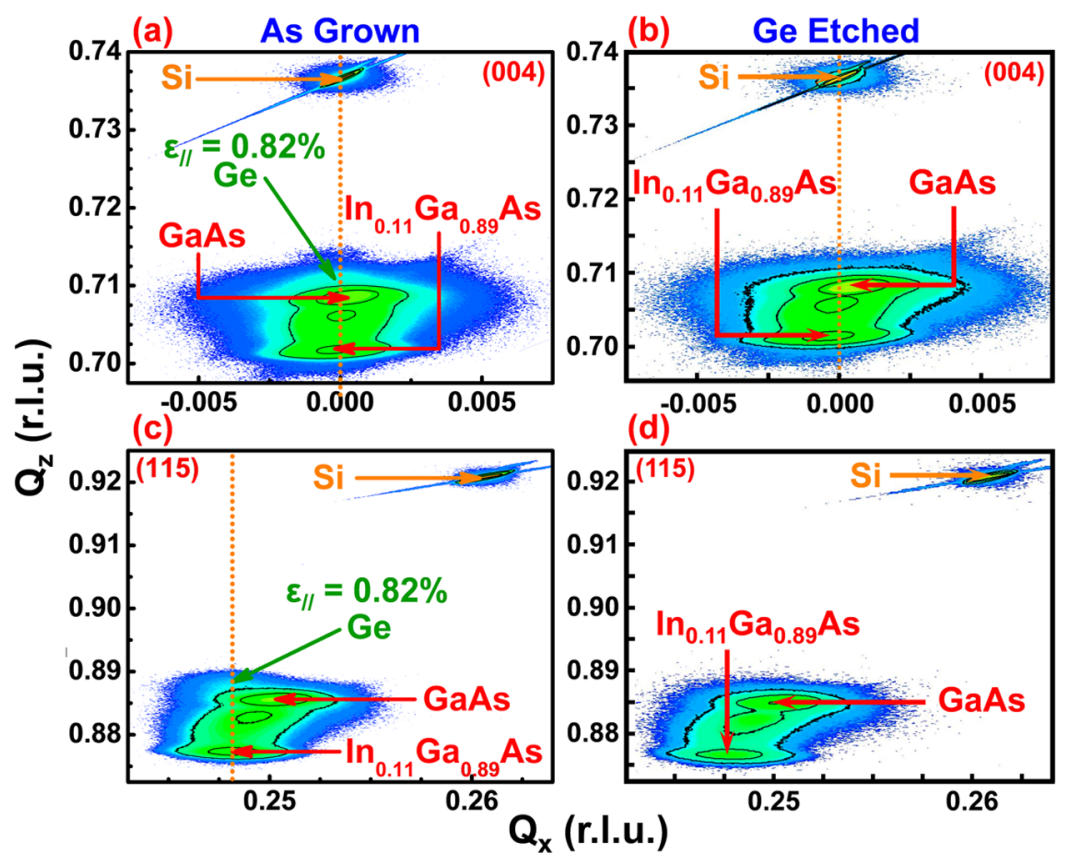

Figure 3. Symmetric (004) RSMs of the $0.82 \% \varepsilon$-Ge/ $\mathrm{In}_{0.11} \mathrm{Ga}_{0.89}$ As heterostructure (a) as-grown and (b) after wet etch removal of the $\varepsilon$-Ge epilayer, highlighting the position of the $\varepsilon$-Ge RLP (a) with respect to the background intensity of the $\operatorname{In}_{0.11} \mathrm{Ga}_{0.89} \mathrm{As} / \mathrm{GaAs}$ metamorphic buffer (b). Panels $\mathrm{c}$ and $\mathrm{d}$ show the same for the asymmetric (115) RSMs. Moreover, the Si RLP is clearly visible.

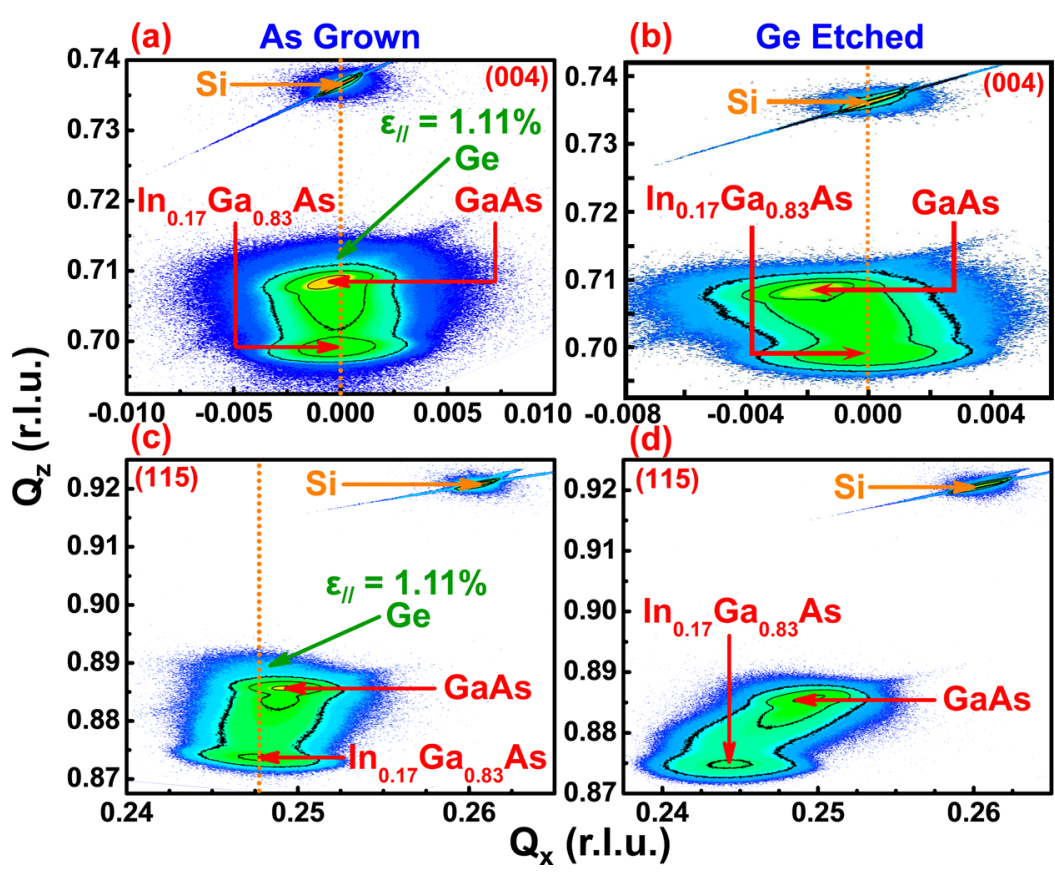

Figure 4. Symmetric (004) RSMs of the $1.11 \% \varepsilon$-Ge/ $\operatorname{In}_{0.17} \mathrm{Ga}_{0.83} \mathrm{As}$ heterostructure (a) following the $\operatorname{In}_{0.17} \mathrm{Ga}_{0.83}$ As cap layer growth and (b) after removal of the top $\operatorname{In}_{0.17} \mathrm{Ga}_{0.83}$ As and $\varepsilon$-Ge epilayers by wet etching, emphasizing the position of the $\varepsilon$-Ge RLP (a) with respect to the background intensity of the $\mathrm{In}_{0.17} \mathrm{Ga}_{0.83} \mathrm{As} / \mathrm{GaAs}$ metamorphic buffer (b). Panels $\mathrm{c}$ and $\mathrm{d}$ show a similar comparison for the asymmetric (115) RSMs taken from the same $\operatorname{In}_{0.17} \mathrm{Ga}_{0.83} \mathrm{As} / \varepsilon$-Ge/ $\mathrm{In}_{0.17} \mathrm{Ga}_{0.83} \mathrm{As}$ double heterostructure.

Also shown in Figures 3 and 4 are the (004) and (115) RSMs recorded for each respective metamorphic buffer structure after removal of the $\varepsilon$-Ge $\left(\operatorname{In}_{0.17} \mathrm{Ga}_{0.83} \mathrm{As} / \varepsilon\right.$-Ge $)$ topmost epilayer(s) using dilute $\mathrm{NH}_{4} \mathrm{OH}: \mathrm{H}_{2} \mathrm{O}_{2}: \mathrm{H}_{2} \mathrm{O}$ (2:1:200 volume ratio). For all RSMs, each layer has been labeled to its corresponding reciprocal lattice point (RLP) for clarity. One can find from the symmetric (004) RCs in Figure 2 and RSMs in Figures 3a and 4a that the Ge RLP exhibits a larger Bragg angle as compared to the GaAs buffer RLP, indicating a smaller out of-plane lattice constant and thus the presence of tensile strain in the Ge epilayer. As shown in the inset of Figure 2, because of the larger lattice constant of the $\operatorname{In}_{x} \mathrm{Ga}_{1-x}$ As $(x=0.11,0.17)$ strain template, the in-plane lattice constant of the heteroepitaxial Ge is stretched to accommodate the in-plane lattice constant of the $\mathrm{In}_{x} \mathrm{Ga}_{1-x} \mathrm{As}$ layer, resulting in a biaxial tensile-strained, pseudomorphic Ge epilayer with an expanded in-plane lattice constant (labeled as a) and reduced out-of-plane lattice constant (labeled as c). One can find from Figures 2-4 that 
(a)

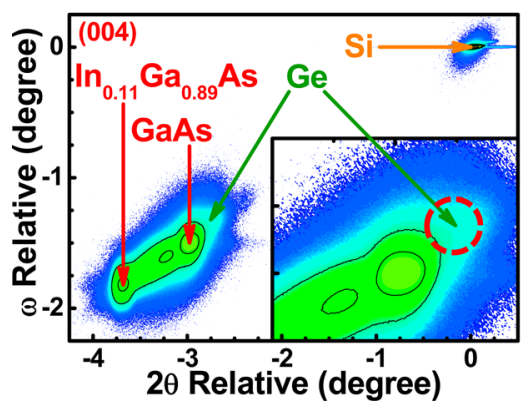

(b) Ge Etched

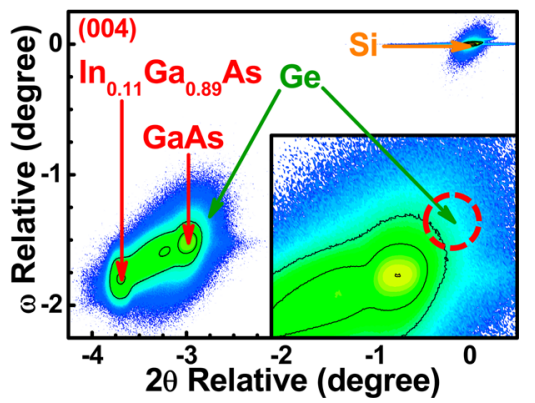

Figure 5. Symmetric (004) RSMs of the $\varepsilon$-Ge/ $\mathrm{In}_{0.11} \mathrm{Ga}_{0.89} \mathrm{As}$ heterostructure (a) as-grown and (b) after etching the $\varepsilon$-Ge epilayer in $\mathrm{NH}_{4} \mathrm{OH}: \mathrm{H}_{2} \mathrm{O}_{2}: \mathrm{H}_{2} \mathrm{O}$, revealing the position of the $\varepsilon$-Ge $\omega-2 \theta$ centroid (a) with respect to the background intensity of the $\mathrm{In}_{0.11} \mathrm{Ga}_{0.89} \mathrm{As} / \mathrm{GaAs}$ metamorphic buffer (b). Insets show magnification of the region in proximity to the GaAS $\omega-2 \theta$ centroid, demonstrating a clear contribution from the $\varepsilon$-Ge diffraction peak to the detected diffraction signal.

Table 1. Summary of the Strain Relaxation Properties of the $\varepsilon$-Ge/ $\operatorname{In}_{x} \mathrm{Ga}_{1-x} \mathrm{As}$ Structures Heterogeneously Integrated onto Si

\begin{tabular}{|c|c|c|c|c|c|c|c|}
\hline \multirow[b]{2}{*}{ material } & \multicolumn{3}{|c|}{ lattice constant $(\AA)$} & \multirow[b]{2}{*}{ In composition (\%) } & \multirow[b]{2}{*}{ relaxation (\%) } & \multirow[b]{2}{*}{ epitaxial tilt (arcsec) } & \multirow[b]{2}{*}{ tensile strain, $\mathrm{Ge}(\%)$} \\
\hline & out-of-plane $\left(a_{\perp}\right)$ & in-plane $\left(a_{\|}\right)$ & relaxed $\left(a_{\mathrm{r}}\right)$ & & & & \\
\hline $\mathrm{In}_{0.11} \mathrm{Ga}_{0.89} \mathrm{As}$ & 5.7073 & 5.6912 & 5.6996 & 11.4 & 82 & -116 & $0.82 \pm 0.06$ \\
\hline $0.82 \% \varepsilon-\mathrm{Ge}$ & 5.6348 & 5.7045 & 5.658 & & & -118 & \\
\hline $\mathrm{In}_{0.17} \mathrm{Ga}_{0.83} \mathrm{As}$ & 5.7286 & 5.7106 & 5.7204 & 16.6 & 87 & 127 & $1.11 \pm 0.03$ \\
\hline $1.11 \% \varepsilon-\mathrm{Ge}$ & 5.6272 & 5.7208 & 5.658 & & & 117 & \\
\hline
\end{tabular}

the diffraction peak and RLP of $\varepsilon$-Ge is partially suppressed by the intensity of the GaAs substrate as a result of minor Bragg angle modulation due to the moderate strain levels studied in this work. To mitigate error in the experimental strain relaxation analysis resulting from the partial superposition of the $\varepsilon$-Ge and GaAs diffraction peaks, only the centroid of the $\varepsilon$ Ge (004) RLP taken from the symmetric (004) RSM was used for quantitative analysis of the out-of-plane lattice spacing. Moreover, so as to more clearly distinguish the superimposed $\varepsilon$-Ge and GaAs peaks, Figure 5 shows the symmetric (004) RSM of the $\varepsilon$-Ge/ $/ \mathrm{In}_{0.11} \mathrm{Ga}_{0.89}$ As structure plotted along $\omega$ - $2 \theta$ (a) prior to and (b) after removal of the $\varepsilon$-Ge layer. As seen in the inset of Figure 5a, magnification of the Bragg angles surrounding the GaAs layer revealed the distinct outline of the $\varepsilon$-Ge peak, thus enabling a more accurate determination of the $\varepsilon$-Ge $\omega-2 \theta$ prior to conversion to reciprocal lattice units. Furthermore, Figures $3 \mathrm{~b}$ and $3 \mathrm{~d}$ ( $4 \mathrm{~b}$ and $4 \mathrm{~d}$ ) show the (004) and (115) RSMs for the $\operatorname{In}_{0.11} \mathrm{Ga}_{0.89} \mathrm{As} / \mathrm{GaAs}\left(\mathrm{In}_{0.17} \mathrm{Ga}_{0.83} \mathrm{As} /\right.$ GaAs) metamorphic buffers following wet etching of the terminating $\varepsilon$-Ge $\left(\operatorname{In}_{0.17} \mathrm{Ga}_{0.83} \mathrm{As} / \varepsilon\right.$-Ge $)$ epilayer(s). One can find by comparing Figures $3 \mathrm{~b}$ and $3 \mathrm{~d}(4 \mathrm{~b}$ and $4 \mathrm{~d})$ to Figures $3 \mathrm{a}$ and $3 c$ ( $4 \mathrm{a}$ and $4 \mathrm{c}$ ) that the $\varepsilon$-Ge RLP indeed contributes significantly to the broadening and intensity of the region in reciprocal space in proximity to the GaAs virtual substrate peak. The ability to clearly differentiate between the $\varepsilon$-Ge and GaAs diffraction peaks thus provides ancillary support for the straindependent shift in the $\varepsilon$-Ge RLP observed in Figures 3 and 4. Further movement of the $\varepsilon$-Ge RLP can be achieved by providing increased tensile strain to the Ge layer through growth on higher indium (In) content $\operatorname{In}_{x} \mathrm{Ga}_{1-x}$ As virtual substrates, thereby increasing the accuracy of the measured strain data.

As shown in Figures $3 \mathrm{c}$ and $4 \mathrm{c}$ by the vertical alignment of the $\operatorname{In}_{x} \mathrm{Ga}_{1-x} \mathrm{As}$ and $\varepsilon$-Ge RLPs (orange dotted lines), the inplane lattice constant of $\varepsilon$-Ge was found to be closely matched with the in-plane lattice constant of the $\operatorname{In}_{0.11} \mathrm{Ga}_{0.89}$ As and $\mathrm{In}_{0.17} \mathrm{Ga}_{0.83} \mathrm{As}$ constant composition layers, signifying that the tensile strain transferred to the Ge was successfully modulated by varying the underlying $\operatorname{In}_{x} \mathrm{Ga}_{1-x}$ As In alloy composition. The detailed analyses of the relaxation and strain states of the epitaxial $\mathrm{Ge}$ and $\operatorname{In}_{x} \mathrm{Ga}_{1-x}$ As layers were evaluated by measuring the in-plane and out-of-plane lattice constants, a and c, for both Ge and $\mathrm{In}_{x} \mathrm{Ga}_{1-x}$ As using the recorded symmetric (004) and asymmetric (115) RSM data. The relaxed lattice constant $\left(a_{\mathrm{r}}\right)$ of each layer was calculated using a, $c$ and Poisson's ratio for each material, noting that a relaxed Ge lattice parameter of $5.658 \AA$ was assumed. ${ }^{32}$ Table 1 summarizes the in-plane, outof-plane, and relaxed lattice constants of the $\operatorname{In}_{x} \mathrm{Ga}_{1-x}$ As $(x=$ $0.11,0.17)$ and $\varepsilon$-Ge layers as well as the amount of strain, relaxation, and epitaxial tilt determined via these measurements. Using the relaxed lattice constant of $\operatorname{In}_{x} \mathrm{Ga}_{1-x} \mathrm{As}$ and Vegard's law, the In alloy composition of each $\operatorname{In}_{x} \mathrm{Ga}_{1-x}$ As virtual substrate was determined to be $11.4 \%$ and $16.6 \%$, which was consistent with the design criteria. The GaAs and $\mathrm{In}_{x} \mathrm{Ga}_{1-x}$ As metamorphic buffers on $\mathrm{Si}$ were found to be $\sim 90 \%$ and $82-$ $87 \%$ relaxed, respectively. The detailed methodology used in the strain relaxation analysis is reported elsewhere. ${ }^{33}$ Additionally, the uncertainty in the experimental strain values for each $\varepsilon$ $\mathrm{Ge} / \mathrm{In}_{x} \mathrm{Ga}_{1-x}$ As heterostructure was derived using the measured effect of epitaxial tilt on the calculated in-plane and out-of-plane lattice parameters and thereby the strain held by the Ge epilayer. For the measured results presented in Table 1 , the data used in the strain relaxation analysis were collected from RSMs in which the tilt of the $\operatorname{In}_{x} \mathrm{Ga}_{1-x}$ As and $\varepsilon$-Ge layers was minimized with respect to the $\mathrm{Si}$ substrate. The resulting uncertainties were found to be $\pm 0.06 \%$ and $\pm 0.03 \%$ for the $0.82 \%$ and $1.11 \% \varepsilon$-Ge, respectively. Consequently, the experimentally demonstrated tensile strain modulation due to increasing In alloy composition in $\operatorname{In}_{x} \mathrm{Ga}_{1-x}$ As strain templates is expected to modify the Ge bandgap, an essential step toward achieving tunable wavelength Ge-based photonic devices, as will be discussed in the microphotoluminescence ( $\mu$-PL) analysis below. 
Strain Analysis via Raman Spectroscopy. For several decades, the semiconductor industry has implemented strain to boost device performance, ${ }^{34,35}$ modify material bandgaps, ${ }^{36}$ and enhance carrier confinement. ${ }^{37}$ Thus, alternative strain measurement techniques to independently confirm material stress have been important technological considerations. Most recently, ${ }^{38}$ Raman spectroscopy has been utilized in determining the $\mathrm{Ge}$ composition in $\mathrm{Si}_{1-x} \mathrm{Ge}_{x}$ layers for source/drain stressors in nanoscale Si CMOS. Figure 6a shows the shift in

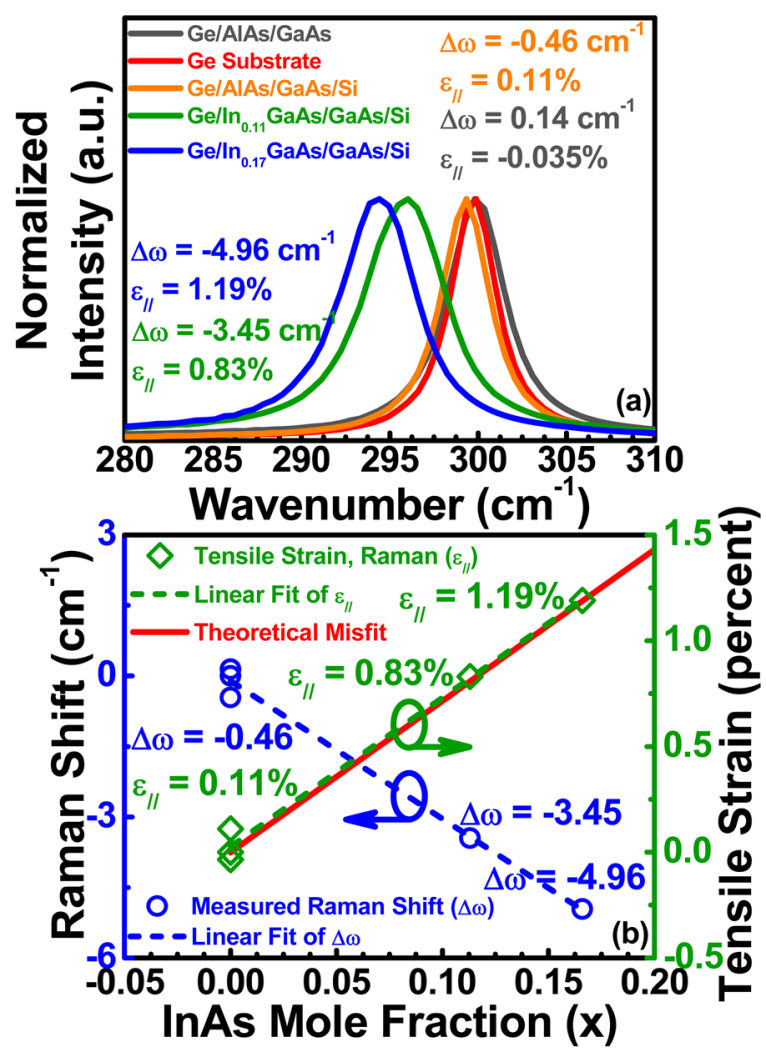

Figure 6. (a) Raman wavenumber shift due to strain-induced modulation of the Ge LO phonon modes. (b) Comparison of Raman-determined strain with wavenumber shift and the theoretical $\mathrm{Ge} / \mathrm{In}_{x} \mathrm{Ga}_{1-x} \mathrm{As}$ misfit.

Raman frequency $(\Delta \omega)$ as a function of strain in the $\varepsilon$-Ge layers studied in this work. As shown in Figure 6a, the tensile strain shifts the position of the longitudinal optical (LO) phonon peak away from the bulk Ge LO peak, where the magnitude and sign of the wavenumber shift (positive/ compressive or negative/tensile) are representative of the type of strain present in the system. The $\varepsilon$-Ge layers grown on Si using a GaAs $/ \mathrm{In}_{x} \mathrm{Ga}_{1-x}$ As graded buffer exhibit wavenumber shifts of -3.45 and $-4.96 \mathrm{~cm}^{-1}$ for the $\varepsilon$-Ge/ $\mathrm{In}_{0.11} \mathrm{Ga}_{0.89}$ As and $\varepsilon-\mathrm{Ge} / \mathrm{In}_{0.17} \mathrm{Ga}_{0.83} \mathrm{As}$ structures, respectively, corresponding to $0.83 \%$ (green) and $1.19 \%$ (blue) tensile strain. Also shown in Figure $6 \mathrm{a}$ is the Raman shift of quasi-lattice matched Ge/AlAs on GaAs (gray) and on Si (orange). It is worth noting that due to the thermal and lattice mismatch present between each epilayer and the growth substrate, some level of residual strain exists in the layers of interest. One can see from Figure 6a that for epitaxial Ge grown quasi-lattice matched to AlAs/GaAs buffers grown on $\mathrm{Si}$, the thermal mismatch between epilayer and substrate results in a tensile strained Ge thin film without the presence of an $\mathrm{In}_{x} \mathrm{Ga}_{1-x}$ As stressor. This thermally induced tensile stress must be accounted for when analyzing the Raman shift of $\varepsilon$-Ge heterogeneously integrated onto Si utilizing III-V buffer architectures. While it is well documented that the $\mathrm{Ge}-$ Ge phonon vibration mode is approximately $300 \mathrm{~cm}^{-1}$ and that a shift in phonon vibration mode with respect to this number is a result of crystallographic strain, an exact relationship between strain and wavenumber shift is only approximate. Recent work $^{39}$ has utilized the relation $\Delta \omega=-b \varepsilon_{\|}$to analyze Raman shift as a function of strain in tensile Ge thin films, where $\Delta \omega$ is the wavenumber shift (in $\mathrm{cm}^{-1}$ ) and $b$ is a material parameter dependent on the material's phononic and elastic constants. Using the reported literature value of $415 \mathrm{~cm}^{-1}$ for $\mathrm{Ge},{ }^{40}$ the wavenumber shift versus In alloy composition as well as strain versus In alloy composition relationships are shown in Figure $6 \mathrm{~b}$. One can find from Figure $6 \mathrm{~b}$ that tensile strain (green) determined from the Raman shift (blue) corresponds accurately to the theoretical misfit (red) for the In alloy compositions studied, thereby independently confirming the pseudomorphic nature of the strained Ge epitaxy. Moreover, the strain analysis demonstrated utilizing Raman spectroscopy was found to be in good agreement with the strain relaxation properties of the $\varepsilon$ $\mathrm{Ge} / \mathrm{In}_{x} \mathrm{Ga}_{1-s} \mathrm{As}$ heterostructures on $\mathrm{Si}$ as determined via X-ray diffraction analysis, thereby highlighting the suitability of Raman spectroscopy for studying $\varepsilon$-Ge thin films with moderate strain levels.

Defect Analysis by TEM. Additional investigation into the material quality and nature of the $\varepsilon$ - $\mathrm{Ge} / \mathrm{In}_{x} \mathrm{Ga}_{1-x}$ As $(x=0.11$, 0.17 ) heterointerface was performed by high-resolution crosssectional transmission electron microscopy (TEM) analysis. Figures $7 \mathrm{a}-\mathrm{f}$ and $8 \mathrm{a}-\mathrm{e}$ show the bright-field cross-sectional TEM micrographs of the $\varepsilon$-Ge grown on $\operatorname{In}_{0.11} \mathrm{Ga}_{0.89} \mathrm{As} /$ $\mathrm{In}_{x} \mathrm{Ga}_{1-x} \mathrm{As} / \mathrm{GaAs} / \mathrm{Si}$ and $\mathrm{In}_{0.17} \mathrm{Ga}_{0.83} \mathrm{As} / \mathrm{In}_{x} \mathrm{Ga}_{1-x} \mathrm{As} / \mathrm{GaAs} / \mathrm{Si}$, respectively. Cross-sectional TEM measurements were performed on various locations of the TEM specimen and the representative results are shown in Figures 7 and 8. Moreover, all layers are labeled in each figure. The GaAs and linearly graded $\operatorname{In}_{x} \mathrm{Ga}_{1-x}$ As buffers on $\mathrm{Si}$ were found to effectively mitigate the lattice mismatch induced defects and dislocations between the $\varepsilon$-Ge $/ \operatorname{In}_{x} \mathrm{Ga}_{1-x} \mathrm{As}(x=0.11,0.17)$ layers of interest and the Si substrate. One can find from Figure $7 \mathrm{a}(8 \mathrm{a})$ that the majority of dislocations were confined within the linearly graded $\operatorname{In}_{x} \mathrm{Ga}_{1-x}$ As buffer. Moreover, the residual strain within the top $\operatorname{In}_{0.11} \mathrm{Ga}_{0.89}$ As $\left(\mathrm{In}_{0.17} \mathrm{Ga}_{0.83}\right.$ As $)$ layer was minimized due to the nominal accommodation of mismatch induced epitaxial strain via misfit dislocation formation and subsequent dislocation glide, corroborating the previously discussed strain relaxation properties for each $\operatorname{In}_{x} \mathrm{Ga}_{1-x}$ As virtual substrate. Similarly, the apparent absence of threading dislocation propagation into the $\varepsilon$-Ge active region suggests a devicequality active region, further reinforcing the structural data found via reciprocal space mapping. Hence, the composite GaAs and linearly graded $\mathrm{In}_{x} \mathrm{Ga}_{1-x}$ As buffer provided a highquality virtual substrate for the tunable tensile-strained $\mathrm{Ge}$ heterogeneously integrated onto Si. Furthermore, high resolution lattice indexing shown in Figure $7 \mathrm{c}(8 \mathrm{~b})$ revealed the lattice line extending uninterrupted between the $\varepsilon$-Ge and $\mathrm{In}_{0.11} \mathrm{Ga}_{0.89} \mathrm{As}\left(\mathrm{In}_{0.17} \mathrm{Ga}_{0.83} \mathrm{As}\right)$ epilayers, indicating that the $\mathrm{Ge}$ in-plane lattice constant was internally registered with the inplane lattice constant of the $\operatorname{In}_{0.11} \mathrm{Ga}_{0.89}$ As $\left(\operatorname{In}_{0.17} \mathrm{Ga}_{0.83} \mathrm{As}\right)$ strain template and demonstrating pseudomorphic tensile-stained $\mathrm{Ge}$ epitaxy in good agreement with the X-ray and Raman analysis presented earlier. Figures $7 \mathrm{~d}-\mathrm{f}$ and $8 \mathrm{c}-\mathrm{e}$ show Fast Fourier Transform (FFT) patterns representative of the regions 

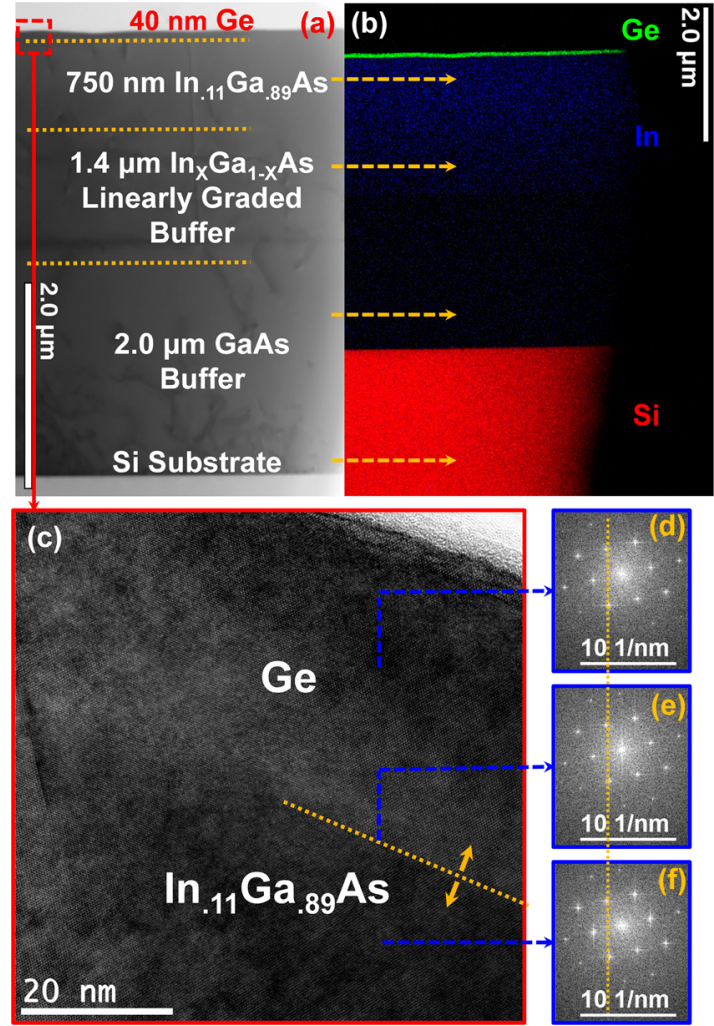

Figure 7. (a) Low-resolution cross-sectional TEM micrograph of the $0.82 \% \varepsilon-\mathrm{Ge} / \mathrm{In}_{0.11} \mathrm{Ga}_{0.89}$ As structure on Si. (b) EDS map of (a) showing distinct, abrupt boundaries between $\varepsilon$-Ge (green)/ $\mathrm{In}_{0.11} \mathrm{Ga}_{0.89}$ As and $\mathrm{GaAs} / \mathrm{Si}$ (red) as well as In grading (blue). (c-f) High-resolution micrographs of the $0.82 \% \quad \varepsilon$-Ge/ $/ \mathrm{In}_{0.11} \mathrm{Ga}_{0.89} \mathrm{As}$ heterointerface and detailed lattice line indexing (including FFT patterns) demonstrating the tensile nature of Ge with respect to the underlying $\mathrm{In}_{0.11} \mathrm{Ga}_{0.89}$ As.

denoted by arrows. The indexing of these FFT patterns indicates that the electron beam was parallel to the [011] orientation. Moreover, the FFT patterns obtained from the $\varepsilon$ Ge layer, $\varepsilon$ - $\mathrm{Ge} / \mathrm{In}_{x} \mathrm{Ga}_{1-x}$ As heterointerface, and the $\operatorname{In}_{x} \mathrm{Ga}_{1-x} \mathrm{As}$ layer are identical and absent of diffraction peak splitting (satellite peaks), indicating the contribution of a singular lattice parameter to the diffraction peak reciprocal spacing in the FFT field-of-view and further validating the high quality, coherent epitaxial growth at the strained $\mathrm{Ge} / \mathrm{In}_{x} \mathrm{Ga}_{1-x} \mathrm{As}$ interface.
Accordingly, the cross-sectional TEM analysis suggests optical-quality film structures with atomically smooth interfaces, which is crucial to minimizing cavity losses in Ge-based photonic devices.

To further evaluate the atomic species profile across each heterointerface, energy dispersive X-ray spectroscopy (EDS) was utilized to perform an elemental mapping of the $\varepsilon$-Ge/ $\mathrm{In}_{0.11} \mathrm{Ga}_{0.89}$ As heterostructure. EDS is a powerful technique that has been extensively used for the analysis of the structural and chemical modulation of nanoscale heterostructures and multilayer heterostructures. ${ }^{41}$ Figure $7 \mathrm{~b}$ shows the elemental mapping of the $\varepsilon$-Ge $/ \mathrm{In}_{0.11} \mathrm{Ga}_{0.89}$ As structure where the $\mathrm{Ge}$ layer (green) was found to be uniformly distributed and the In (blue) exhibits a variable composition, as expected in the linearly graded $\operatorname{In}_{x} \mathrm{Ga}_{1-x}$ As buffer layer, in addition to the composition profile of Si (red). The EDS analysis exhibits a uniform and sharp heterointerface between the $\varepsilon$-Ge and the $\mathrm{In}_{0.11} \mathrm{Ga}_{0.89}$ As layer, with no apparent interdiffusion across any of the structure's heterointerfaces despite the increased thermal budget as a result of experiencing extended growth temperature conditions during the strained layer epitaxy of Ge. Therefore, the atomic scale imaging and microstructural analysis feedback loop is necessary for the large-scale heterointegration of tunable tensile-strained $\mathrm{Ge}$ on $\mathrm{Si}$.

Strain-Induced Ge Bandgap Modulation Analysis via Photoluminescence. Photoluminescence spectroscopy (PL) has been extensively used to investigate strained-induced bandgap shifts, ${ }^{42}$ the competition between direct and indirect emission characteristics, ${ }^{43,44}$ radiative and nonradiative recombination properties, ${ }^{45}$ and the ratio between direct and indirect bandgap recombination with increasing strain in Ge. ${ }^{36}$ In this work, low-temperature micro-PL $(\mu$-PL) measurements were performed at $7.5 \mathrm{~K}$ utilizing an incident power density of 860 $\mathrm{kW} / \mathrm{cm}^{2}$ and excitation wavelength of $800 \mathrm{~nm}$. This high power density is required to obtain a reasonable $\mathrm{PL}$ emission intensity. ${ }^{46,47}$ Figure 9a shows the PL spectra obtained from $0.82 \%$ (blue) and $1.11 \% \varepsilon$-Ge with (red) and without (green) $\mathrm{In}_{0.17} \mathrm{Ga}_{0.83}$ As capping layer as well as quasi-lattice matched $\mathrm{Ge}$ on AlAs/GaAs (gray). The PL spectra have been shifted vertically for clarity. One can see from Figure 9a that the main peak position is red-shifted toward lower photon energies (higher wavelengths) due to the increase in tensile strain and corresponding reduction in $\varepsilon$-Ge bandgaps. Furthermore, from Figure $9 \mathrm{~b}$ and $\mathrm{c}$, one can see that each sample exhibits a convolved emission spectra containing a single, intense peak
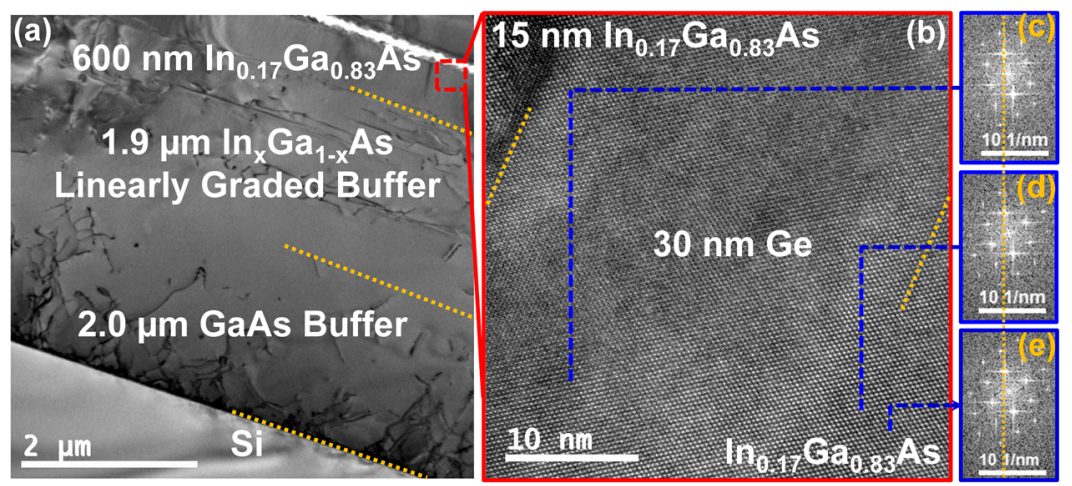

Figure 8. (a) Low-resolution cross-sectional TEM micrograph of the $1.11 \% \varepsilon$-Ge/ $/ \mathrm{In}_{0.17} \mathrm{Ga}_{0.83} \mathrm{As}$ structure on $\mathrm{Si}$. (b-e) High-resolution micrograph of the $1.11 \% \mathrm{In}_{0.17} \mathrm{Ga}_{0.83} \mathrm{As} / \varepsilon$ - $\mathrm{Ge} / \mathrm{In}_{0.17} \mathrm{Ga}_{0.83}$ As heterointerfaces and detailed lattice line indexing (including FFT patterns) demonstrating the tensile nature of $\mathrm{Ge}$ with respect to the underlying $\mathrm{In}_{0.17} \mathrm{Ga}_{0.83} \mathrm{As}$. 


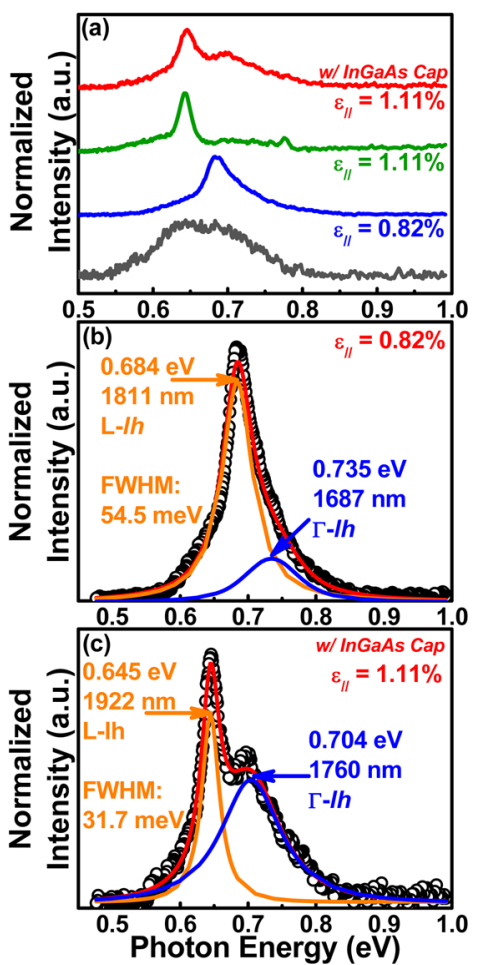

Figure 9. (a) Red-shift (plotted in photon energy) due to strain in the $\varepsilon$-Ge epilayers as measured by $\mu$-PL at $7.5 \mathrm{~K}$. (b and c) GaussLorentzian fitting of the $\varepsilon-\mathrm{Ge} / \mathrm{In}_{0.11} \mathrm{Ga}_{0.89} \mathrm{As}$ (b) and $\varepsilon$-Ge/ $\mathrm{In}_{0.17} \mathrm{Ga}_{0.83}$ As (c) emission.

and a shoulder whose relative intensity is strongly straindependent.

The deconvolution of these spectral features into accurate transition energies requires considering several coinciding factors, most importantly measurement temperature and strain incorporation. Although it has been reported that experimental collection geometries normal to the sample surface (i.e., $\hat{z}$ ) favor conduction band coupling with the heavy-hole (hh) valence band, ${ }^{36}$ cryogenic sample temperatures result in a rapid depopulation of the hh valence band due to its higher energy states. Consequently, radiative recombination between the conduction band minima and light-hole (lh) valence band increases as heavy-holes undergo rapid nonradiative relaxation into lower energy states within the lh valence band. The dominance of the L-lh and $\Gamma$-lh coupling at low measurement temperatures is seen experimentally through direct observation of the lower energy transitions, i.e. measurement of the lower optical energy gaps within a material, which were indeed detected in the measured $\mu$-PL spectra in Figures $7 \mathrm{~b}$ and $7 \mathrm{c}$. Nevertheless, a disparity in intensity between the deconvolved L-lh and $\Gamma$-lh transitions exists, which is in contrast to the reported $^{43,44,46-49}$ nature of the competitiveness between direct and indirect optical transitions in $\mathrm{Ge}$ as a function of temperature. This can be explained via the compounding effects of strain-dependent gain enhancement, prohibitively large energy separations between the $\mathrm{L}$ and $\Gamma$ conduction band minima, and momentum contribution to the indirect L-lh recombination path from exciton-generated longitudinal acoustic (LA) phonons. In the former case, several theoreti$\mathrm{cal}^{50-53}$ and experimental ${ }^{54,55}$ studies have demonstrated the effects of increasing tensile strain and doping concentrations on optical gain (or absorption) in Ge films. In particular, Virgilio et al. concluded that for a fixed optical pumping power, the absorption spectrum minimum (i.e., $\alpha(\hbar \omega)$ ) at the sample's surface trends approximately linearly toward lower photon energies as biaxial tensile strain increases. ${ }^{50}$ The wavelengthdependence of the absorption coefficient in Ge films as a function of strain proposed by Virgilio and co-workers is found to be in good agreement with prior experimental results ${ }^{54,55}$ investigating the temperature- and strain-dependence of Ge optical absorption. From these previous results, one would expect that the lower energy spectral features would exhibit higher relative PL intensities when compared with the higher energy features, which is observed in Figures $9 \mathrm{~b}$ and 9c. Nonetheless, one might anticipate that due to the lowtemperature nature of the sample during optical characterization, $\Gamma$ valley recombination would dominate due to reduced phonon-assisted momentum conservation between the L- and $\Gamma$-points in momentum space. This interpretation, however, fails to address the need for additional energy in order to surmount the $\mathrm{L}-\Gamma$ conduction band minima separation, which is in addition to the required momentum preservation. For unstrained, bulk Ge, the separation between the $\mathrm{L}$ and $\Gamma$ minima is approximately $150 \mathrm{meV}$. Thus, although momentum conservation may be met via exciton-generated LA phonons at cryogenic measurement temperatures, ${ }^{56-58}$ the aforementioned $\mathrm{L}-\Gamma$ energy difference restricts radiative recombination primarily to $\mathrm{L}$ valley pathways for low-to-moderate strains and doping concentrations in which the $\mathrm{L}-\Gamma$ separation remains prohibitively large. It is worth noting that previous work ${ }^{59}$ investigating similar $\varepsilon$ - $\mathrm{Ge} / \mathrm{In}_{x} \mathrm{Ga}_{1-x}$ As double heterostructures revealed an integrated PL intensity power-dependence suggestive of exciton recombination for type-I $\varepsilon$-Ge/ $\mathrm{In}_{x} \mathrm{Ga}_{1-x}$ As heterointerfaces. The detailed analysis of the heterointerface energy band alignment for the structures studied in this work can be found in a subsequent section, suffice to say the structures indeed exhibited a type-I band alignment, thus reinforcing the notion that exciton-phonon interaction underlies the observed indirect radiative recombination. ${ }^{60}$ Moreover, as higher tensile strains are incorporated, the more rapid lowering of the $\Gamma$ valley minimum results in a similarly rapid decrease in the $\mathrm{L}-\Gamma$ separation energy, thereby enhancing direct gap recombination as validated by the increase in relative intensity and integrated peak area of the $\Gamma-\mathrm{lh}$ transition between Figure $9 \mathrm{~b}$ and $\mathrm{c}$.

Lastly, it is generally understood that the reduction in effective mass in the lh valence band due to strain correlates with a decreasing density of states and an increasingly restricted range of transition energies. Hence, it is expected that the fullwidth at half-maximum (fwhm) of the more prominent $\mathrm{L}-\mathrm{lh}$ transition as well as the ratio of the integrated peak areas between $\mathrm{L}-\mathrm{lh}$ and $\Gamma-\mathrm{lh}$ transitions (i.e., $I_{\mathrm{L}-\mathrm{lh}}: I_{\Gamma-\mathrm{lh}}$ ) will both decrease as the biaxial tensile strain held by the Ge epilayer increases. Figures $8 \mathrm{~b}$ and $\mathrm{c}$ demonstrate that for increasing tensile strain, the fwhm of the $\mathrm{L}-\mathrm{lh}$ transition indeed decreases from $54.5 \mathrm{meV}$ at $\varepsilon=0.82 \%$ to $31.7 \mathrm{meV}$ at $\varepsilon=1.11 \%$. Likewise, the ratio of the $\mathrm{L}-\mathrm{lh}$ to $\Gamma-\mathrm{lh}$ integrated peak areas also decreases from 1.95 at $\varepsilon=0.82 \%$ to 0.30 at $\varepsilon=1.11 \%$, indicating that the increase in biaxial tensile strain strongly enhances $\Gamma-\mathrm{lh}$ recombination via significant $\Gamma-\mathrm{lh}$ bandgap reduction as well as restricts the energy range for $\mathrm{L}-\mathrm{lh}$ radiative recombination. Figure 10 compares the experimentally observed bandgaps (symbols) and strain determined from $\mu$ PL and XRD analysis, respectively, with the predicted dependence of the low-temperature Ge bandgap on increasing 


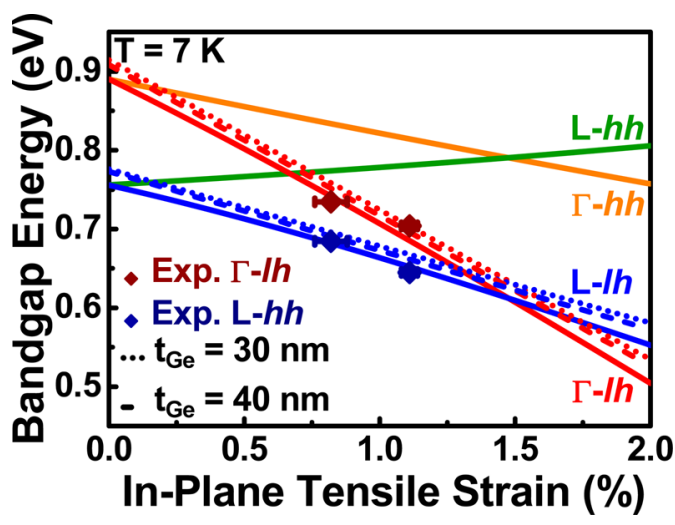

Figure 10. $\mu$-PL determined bandgaps of $0.82 \%$ and $1.11 \% \varepsilon$-Ge in comparison to the theoretical bandgap-strain dependence for $\mathrm{Ge}$ calculated using a $30 \times 30 \mathrm{k} \cdot \mathrm{p}$ model taking into quantization-induced bandgap enhancement at decreased $\varepsilon$-Ge layer thicknesses. Good agreement is found between the measured (symbols) and predicted (lines) bandgaps as a function of increasing tensile strain.

strain (solid lines) calculated using a $30 \times 30 \mathbf{k} \cdot \mathbf{p}$ formalism. ${ }^{61}$ Additionally, the effect of decreasing $\varepsilon$-Ge epilayer thickness $\left(t_{\mathrm{Ge}}\right)$ on the $\varepsilon$-Ge bandgap because of energy level quantization is shown via the dashed $\left(t_{\mathrm{Ge}}=40 \mathrm{~nm}\right)$ and dotted $\left(t_{\mathrm{Ge}}=30\right.$ $\mathrm{nm})$ lines. The measured $\mathrm{L}-\mathrm{lh}(\Gamma-\mathrm{lh})$ bandgaps were found to be $0.684(0.735 \mathrm{eV})$ and $0.645 \mathrm{eV}(0.704 \mathrm{eV})$ for the $\varepsilon$-Ge/ $\mathrm{In}_{0.11} \mathrm{Ga}_{0.89} \mathrm{As}$ and $\varepsilon$-Ge $/ \mathrm{In}_{0.17} \mathrm{Ga}_{0.83}$ As structures, respectively. This is in excellent agreement with the theoretical bandgapstrain relation shown in Figure 10, noting that the quantization effect induced from decreasing $\varepsilon$-Ge layer thickness has a negligible impact on the measured bandgaps for the $\varepsilon$-Ge film thicknesses studied in this work. Furthermore, the demonstration of tunable wavelength $\varepsilon$-Ge epitaxial layers heteroge- neously integrated onto $\mathrm{Si}$ is a key first step toward the monolithic integration of Ge-based photonic devices and optically active layers with state-of-the-art CMOS technology as well as the development of energy-efficient light sources for future on-chip optical interconnects.

Energy Band Offset Determination via XPS. Understanding of the energy band alignment between $\varepsilon$-Ge and the underlying constant composition $\operatorname{In}_{x} \mathrm{Ga}_{1-x}$ As layer is essential for evaluating carrier confinement in future $\varepsilon$-Ge-based optical devices on $\mathrm{Si}$. The valence band and conduction band offsets at the $\varepsilon$ - $\mathrm{Ge} / \mathrm{In}_{x} \mathrm{Ga}_{1-x}$ As heterointerface were determined using $\mathrm{X}$ ray photoelectron spectroscopy (XPS) by measuring the atomic core level ( $\mathrm{CL}$ ) binding energies in $\varepsilon$-Ge ( $\mathrm{Ge} 3 \mathrm{~d}$ ) and $\mathrm{In}_{x} \mathrm{Ga}_{1-x}$ As (As $3 \mathrm{~d}_{5 / 2}$ ), the valence band maxima (VBM) of each material, and the interfacial CL binding energy shifts in each material. In order to accurately determine the band offsets, three samples were selected for XPS analysis: (i) $40(30 \mathrm{~nm}) \varepsilon$ Ge on $\operatorname{In}_{0.11} \mathrm{Ga}_{0.89}$ As $\left(\operatorname{In}_{0.17} \mathrm{Ga}_{0.83}\right.$ As $)$ was used to measure the $\mathrm{CL}$ and VBM binding energy spectra for $\varepsilon$-Ge; (ii) $750 \mathrm{~nm}$ $(600 \mathrm{~nm}) \mathrm{In}_{0.11} \mathrm{Ga}_{0.89}$ As $\left(\operatorname{In}_{0.17} \mathrm{Ga}_{0.83} \mathrm{As}\right)$ without the top $\varepsilon$-Ge layer was used to measure the $\mathrm{CL}$ binding energy of arsenic (As) and the VBM of $\operatorname{In}_{0.11} \mathrm{Ga}_{0.89}$ As $\left(\operatorname{In}_{0.17} \mathrm{Ga}_{0.83} \mathrm{As}\right.$ ); and (iii) $\sim 2 \mathrm{~nm} \quad \mathcal{E}$-Ge/ $/ \mathrm{In}_{0.11} \mathrm{Ga}_{0.89}$ As $\left(\operatorname{In}_{0.17} \mathrm{Ga}_{0.83} \mathrm{As}\right)$ was used to measure the $\mathrm{CL}$ binding energy shifts of Ge and As at the $\varepsilon$ $\mathrm{Ge} / \mathrm{In}_{x} \mathrm{Ga}_{1-x}$ As heterointerface. The binding energy was corrected by adjusting the carbon (C) 1s CL peak position to $285.0 \mathrm{eV}$ for each sample surface. Residual native oxide was removed from the surface of $\mathrm{Ge}$ and $\mathrm{In}_{x} \mathrm{Ga}_{1-x}$ As using dilute $\mathrm{NH}_{4} \mathrm{OH}: \mathrm{H}_{2} \mathrm{O}_{2}: \mathrm{H}_{2} \mathrm{O}$ (2:1:200 volume ratio) prior to loading into the XPS chamber. The valence band offset $\left(\Delta E_{\mathrm{v}}\right)$ between the $\varepsilon$-Ge and the $\operatorname{In}_{x} \mathrm{Ga}_{1-x}$ As layer was determined using Kraut's method, ${ }^{62}$ which is widely used in the analysis of heterointerface band discontinuities at semiconductor/semi-

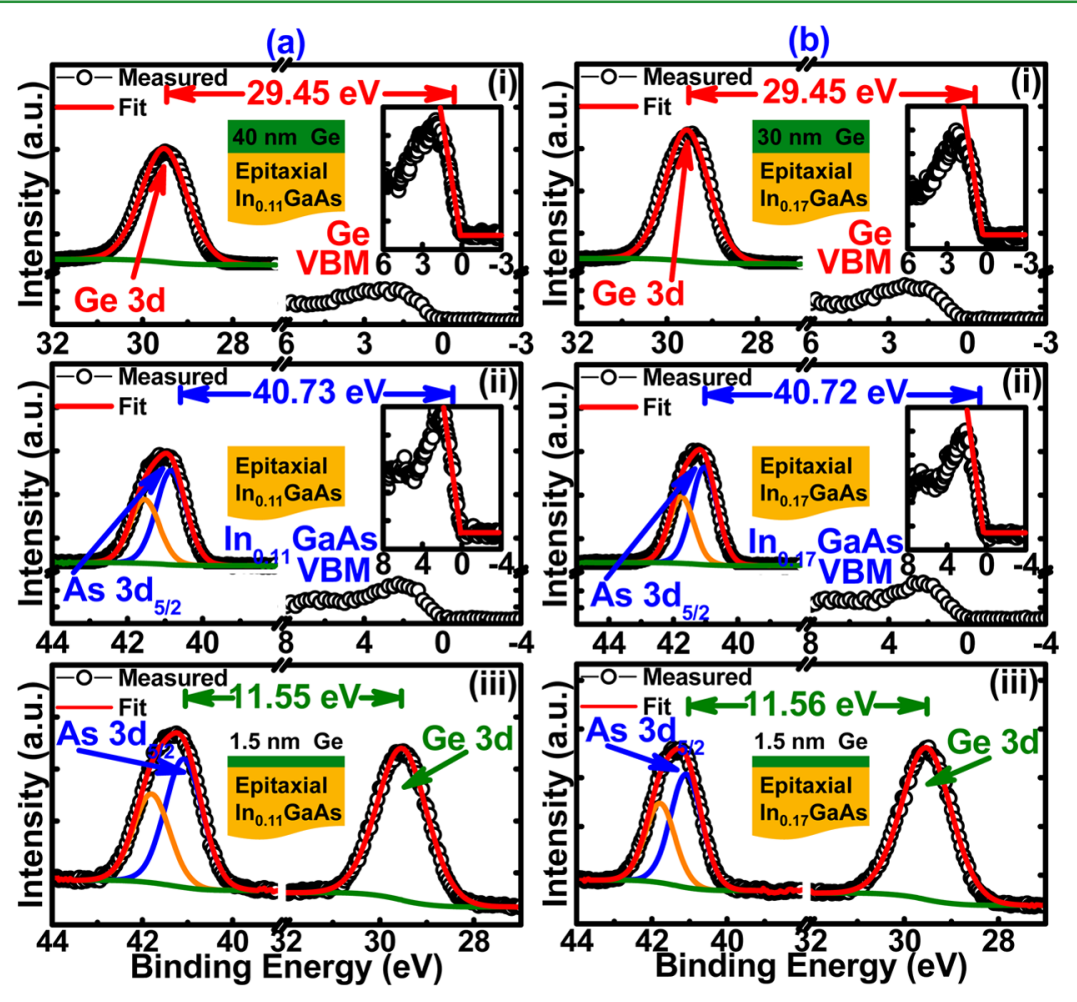

Figure 11. Representative photoelectron spectra used in the valence band analysis for the $\varepsilon$-Ge/ $\operatorname{In}_{0.11} \mathrm{Ga}_{0.89} \mathrm{As}(\mathrm{a})$ and $\varepsilon$-Ge/In $0.17 \mathrm{Ga} 0.83 \mathrm{As}(\mathrm{b})$ structures, corresponding to (i) thick (>10 nm) $\varepsilon$-Ge emission, (ii) bulk $\operatorname{In}_{x} \mathrm{Ga}_{1-x}$ As emission, and (iii) interfacial core level binding energy shifts. 
conductor $^{63-66}$ as well as dielectric/semiconductor ${ }^{67,68}$ heterojunctions. The positive charges generated during the XPS measurements were neutralized by flowing electrons through the sample stage to prevent uncompensated electron loss that could potentially affect the interfacial band bending results due to sample charging. The valence band offset can be written as ${ }^{62}$

$$
\begin{aligned}
\Delta E_{\mathrm{V}}= & \left(E_{\mathrm{Ge} 3 \mathrm{~d}}^{\mathrm{Ge}}-E_{\mathrm{VBM}}^{\mathrm{Ge}}\right)-\left(E_{\mathrm{As} 3 \mathrm{~d}_{5 / 2}}^{\mathrm{InGaAs}}-E_{\mathrm{VBM}}^{\mathrm{InGaAs}}\right) \\
& +\left(E_{\mathrm{As}_{\mathrm{d}} \mathrm{d}_{5 / 2}}^{\mathrm{InGaAs}}(i)-E_{\mathrm{Ge} 3 \mathrm{~d}}^{\mathrm{Ge}}(i)\right)
\end{aligned}
$$

where $E_{\mathrm{Ge} 3 \mathrm{~d}}^{\mathrm{Ge}}$ and $E_{\mathrm{As} 3 \mathrm{~d}_{5 / 2}}^{\mathrm{InGAs}}$ are the CL binding energies of $\varepsilon$-Ge Ge $3 \mathrm{~d}$ and $\operatorname{In}_{x} \mathrm{Ga}_{1-x}$ As As $3 \mathrm{~d}_{5 / 2}$ CLs, respectively, and $E_{\mathrm{VBM}}$ is the $\mathrm{VBM}$ of the corresponding material. $E_{\mathrm{VBM}}$ is determined by linearly fitting the leading edge of the valence band (VB) spectra to the photoemission background line. $E_{\mathrm{As}_{3} \mathrm{~d}_{5 / 2}}^{\mathrm{InGaAs}}(i)$ and $E_{\mathrm{Ge} 3 \mathrm{~d}}^{\mathrm{Ge}}(i)$ are the $\mathrm{CL}$ binding energies of $\mathrm{As} 3 \mathrm{~d}_{5 / 2}$ and $\mathrm{Ge} 3 \mathrm{~d}$ measured at the heterointerface. The conduction band offset $\left(\Delta E_{\mathrm{c}}\right)$ can be estimated by ${ }^{63-68}$

$$
\Delta E_{\mathrm{C}}=E_{\mathrm{G}}^{\mathrm{InGaAs}}-\Delta E_{\mathrm{V}}-E_{\mathrm{G}}^{\mathrm{Ge}}
$$

where $E_{\mathrm{G}}^{\mathrm{InGaAs}}$ and $E_{\mathrm{G}}^{\mathrm{Ge}}$ are the bandgap energies of $\mathrm{In}_{0.11} \mathrm{Ga}_{0.89} \mathrm{As}$ $\left(\mathrm{In}_{0.17} \mathrm{Ga}_{0.83} \mathrm{As}\right)$ and $\varepsilon$-Ge, respectively. The $\varepsilon$-Ge bandgap was extracted from $\mu$-PL measurements as discussed above and assumes the experimentally determined $\Gamma$-lh bandgap in calculating $\Delta E_{\mathrm{C}}$, whereas the bandgap energy of $\mathrm{In}_{x} \mathrm{Ga}_{1-x}$ As $(x=0.11,0.17)$ was estimated from ref 69 . Figures $11 \mathrm{a}-\mathrm{b}$ show the CL and VB spectra from (i) $\varepsilon$-Ge, (ii) $\operatorname{In}_{0.11} \mathrm{Ga}_{0.89}$ As and $\mathrm{In}_{0.17} \mathrm{Ga}_{0.83} \mathrm{As}$, and (iii) the $\varepsilon$-Ge/ $\mathrm{In}_{0.11} \mathrm{Ga}_{0.89} \mathrm{As}$ and $\varepsilon$-Ge/ $\mathrm{In}_{0.17} \mathrm{Ga}_{0.83} \mathrm{As}$ (b) interfaces, respectively. The inset shows the schematic layer diagram of the sample used for each measurement. From these spectra, the values of $E_{\mathrm{Ge} 3 \mathrm{~d}}^{\mathrm{Ge}}-E_{\mathrm{VBM}}^{\mathrm{Ge}}$, ${ }_{\mathrm{As} s \mathrm{~d}_{5 / 2}}^{\mathrm{InGaAs}}-E_{\mathrm{VBM}}^{\mathrm{InGaAs}}$, and $E_{\mathrm{As} 3 \mathrm{~d}_{5 / 2}}^{\mathrm{InGSAs}}(i)-E_{\mathrm{Ge} 3 \mathrm{~d}}^{\mathrm{Gn}}(i)$ were determined to be $29.45,40.73$, and $11.55 \mathrm{eV}$, respectively, for the $\varepsilon$-Ge/ $\mathrm{In}_{0.11} \mathrm{Ga}_{0.89} \mathrm{As}$ structure and 29.45, 40.72, and $11.56 \mathrm{eV}$ for the $\varepsilon-\mathrm{Ge} / \mathrm{In}_{0.17} \mathrm{Ga}_{0.83}$ As structure. $\Delta E_{\mathrm{v}}$ was determined to be $0.27 \pm 0.05 \mathrm{eV}(0.29 \pm 0.05 \mathrm{eV})$ using eq 1 and the measured binding energy differences. The uncertainty value is taken from the scattering of the VB data with respect to the linear fitting of the VBM position. $\Delta E_{\mathrm{c}}$ was determined using eq 2 and the bandgap energy of intrinsic $\operatorname{In}_{0.11} \mathrm{Ga}_{0.89}$ As $\left(\operatorname{In}_{0.17} \mathrm{Ga}_{0.83} \mathrm{As}\right)$ at $300 \mathrm{~K}$, that is, $1.26 \mathrm{eV}(1.19 \mathrm{eV}){ }^{69}$ The tensile-strain modified Ge $\Gamma$-lh bandgap energies of $\sim 0.70$ and $\sim 0.73 \mathrm{eV}$ were determined from $\mu$-PL measurements and resulted from the simultaneous lifting of the light-hole/heavy-hole valence band degeneracy and reduction of the $\Gamma$-point conduction band minima. Using these data, $\Delta E_{\mathrm{c}}$ was calculated to be $0.26 \pm 0.1$ $\mathrm{eV}(0.20 \pm 0.1 \mathrm{eV})$. It is worth noting that the fitting of the $\Gamma$ lh bandgap energy from the experimental $\mu$-PL spectra becomes less precise at lower strain levels due to the decreased intensity of the $\Gamma$-lh optical transition (see $\mu$-PL analysis section), resulting in an increased convolution between the L-lh and $\Gamma$-lh emission spectra and therefore increased error in the calculated $\Delta E_{\mathrm{c}}$ value. Additionally, the temperature dependence of the optical bandgaps in Ge will correlate to an increase in $\Delta E_{\mathrm{c}}$ as a function of increasing temperature because of the reduction in the Ge bandgap. Hence, the calculated $\Delta E_{\mathrm{c}}$ values reported here provide guidance on the effect of increasing tensile strain at the $\varepsilon$-Ge $/ \operatorname{In}_{x} \mathrm{Ga}_{1-x}$ As heterointerface, however are associated with increased uncertainty as compared to the measured $\Delta E_{\mathrm{v}}$ values.
Figure 12 shows the schematic band alignment diagrams for the (a) $\varepsilon$-Ge $/ \mathrm{In}_{0.11} \mathrm{Ga}_{0.89} \mathrm{As}$ and (b) $\varepsilon$-Ge/ $/ \mathrm{In}_{0.17} \mathrm{Ga}_{0.83} \mathrm{As}$

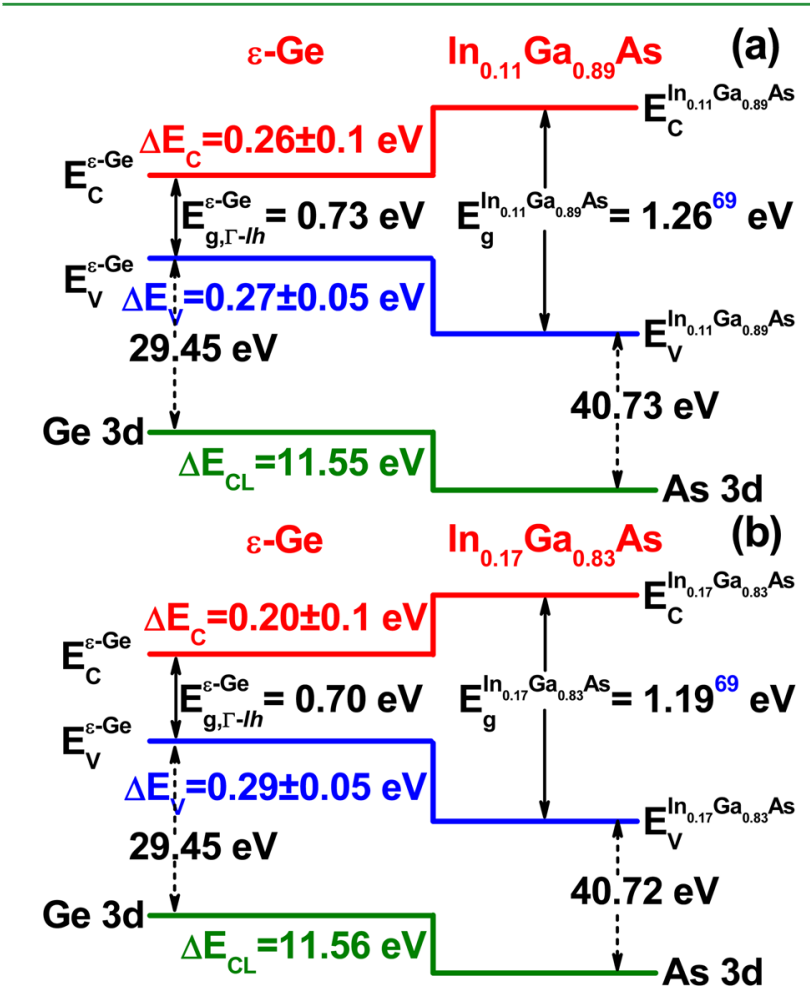

Figure 12. Experimentally determined band alignments for the $\varepsilon$-Ge/ $\mathrm{In}_{0.11} \mathrm{Ga}_{0.89}$ As (a) and $\varepsilon$-Ge/ $\mathrm{In}_{0.17} \mathrm{Ga}_{0.83}$ As (b) heterointerfaces utilizing the measured $\Gamma$-lh bandgaps.

heterojunctions based on the results presented above. One can find from Figure 12 that the band alignment at the $\varepsilon$-Ge/ $\mathrm{In}_{x} \mathrm{Ga}_{1-x} \mathrm{As}$ heterojunction is type-I for the strain states investigated in this work, which is essential for carrier confinement in the $\varepsilon$-Ge layer for group-IV-based photonic devices. Moreover, the shift in $\Delta E_{\mathrm{V}}$ as a function of increasing tensile strain in Ge and In alloy composition in the underlying strain template corroborates the trend previously reported for low-to-high strain $\varepsilon$ - $\mathrm{Ge} / \operatorname{In}_{x} \mathrm{Ga}_{1-x}$ As heterojunctions grown on GaAs substrates, ${ }^{63}$ indicative of a successful integration scheme for tensile-strained Ge active layers on III-V metamorphic buffer architectures implemented on Si.

\section{CONCLUSIONS}

In summary, tunable tensile-strained $\mathrm{Ge}$ heterogeneously integrated on $\mathrm{Si}$ using composite GaAs and linearly graded $\mathrm{In}_{x} \mathrm{Ga}_{1-x}$ As buffers grown by solid source molecular beam epitaxy opens the opportunity for strain-engineered group-IVbased photonic devices on Si. High-resolution X-ray diffraction provided the strain state of each $\varepsilon$-Ge layer as well as the micro structural quality of the Ge thin films and buffer architectures. The tensile strain was further corroborated by micro-Raman spectroscopy, where the strain induced peak shift with respect to bulk $\mathrm{Ge}$ validated the strain state of the $\varepsilon$-Ge. Sharp heterointerfaces between each $\varepsilon$-Ge epilayer and the respective $\mathrm{In}_{x} \mathrm{Ga}_{1-x}$ As virtual substrate were achieved, as demonstrated by high-resolution cross-sectional transmission electron microscopy. Low-temperature microphotoluminescence $(\mu$-PL) measurements demonstrated optical transitions from the $\mathrm{L}$ and $\Gamma$ valley conduction bands to the light-hole (lh) valence band for 
all $\varepsilon$-Ge strain states studied in this work. Furthermore, the $\mu$ $\mathrm{PL}$ measurements demonstrated an effective, strain-induced bandgap modulation, with L-lh $(\Gamma-\mathrm{lh})$ bandgaps of $0.684 \mathrm{eV}$ $(0.735 \mathrm{eV})$ and $0.645 \mathrm{eV}(0.704 \mathrm{eV})$ for the $0.82 \pm 0.06 \%$ and $1.11 \pm 0.03 \%$ strained Ge layers, respectively. A type-I energy band alignment with valence band offsets of 0.27 and $0.29 \mathrm{eV}$ for the $\varepsilon$-Ge/ $\mathrm{In}_{0.11} \mathrm{Ga}_{0.89} \mathrm{As}$ and $\varepsilon$-Ge/ $\mathrm{In}_{0.17} \mathrm{Ga}_{0.83}$ As heterojunctions were demonstrated via $\mathrm{X}$-ray photoelectron spectroscopy analysis, suggesting that the $\varepsilon$ - $\mathrm{Ge} / \mathrm{In}_{x} \mathrm{Ga}_{1-x}$ As heterointerface is feasible for carrier confinement in optical devices utilizing increased strain. This is the first experimental and comprehensive study of strain-engineered tensile-strained epitaxial Ge layers heterogeneously integrated on Si. Consequently, the tunable tensile-strained $\mathrm{Ge}$ materials growth, structural and optical properties, and band offset parameters pave the way for the realization of novel Ge-based photonic devices integrated on $\mathrm{Si}$.

\section{MATERIALS AND METHODS}

Material Synthesis. Unintentionally doped tensile-strained epitaxial 30-40 nm thick Ge layers were grown on off-cut (100) Si substrates using an in situ growth process utilizing separate solidsource molecular beam epitaxy (MBE) growth chambers for the Ge and III-V materials, connected via an ultrahigh vacuum transfer chamber. The effect of substrate off-cut on the suppression of antiphase domain boundary and stacking fault formation at the GaAs/ $\mathrm{Si}$ interface is well-supported in the literature ${ }^{70}$ and was utilized to achieve device quality active layers in this work. GaAs and linearly graded $\mathrm{In}_{x} \mathrm{Ga}_{1-x}$ As buffers were grown on $\mathrm{Si}$ to mitigate the defects and dislocations due to the significant lattice and thermal mismatch between $\mathrm{Ge}$ and Si. An $\mathrm{In}_{0.11} \mathrm{Ga}_{0.89}$ As or $\mathrm{In}_{0.17} \mathrm{Ga}_{0.83}$ As constant composition virtual substrate was grown prior to Ge layer growth, immediately followed by strained-layer $\mathrm{Ge}$ epitaxy at a growth temperature of $400{ }^{\circ} \mathrm{C}$ and growth rate of $\sim 0.1 \AA / s$, respectively. During the substrate oxide desorption and periodically throughout each layer growth, reflection high energy electron diffraction (RHEED) was used to monitor the surface reconstruction for growth-induced defect patterns. After the $\mathrm{GaAs} / \mathrm{In}_{x} \mathrm{Ga}_{1-x} \mathrm{As}$ layer growth, the sample was transferred under ultrahigh vacuum to the Ge MBE chamber for subsequent $\varepsilon$-Ge growth. The $\varepsilon$-Ge on Si samples were characterized by high-resolution $\mathrm{X}$-ray diffraction, atomic force microscopy, and low-temperature magneto-transport measurements. Select samples were then transferred to the III-V chamber for the upper $\operatorname{In}_{x} \mathrm{Ga}_{1-x}$ As layer growth. Prior to the $\operatorname{In}_{x} \mathrm{Ga}_{1-x}$ As cap layer growth, samples were held at $400{ }^{\circ} \mathrm{C}$ for $30 \mathrm{~min}$ to thermally desorb residual Ge surface oxides formed during ex-situ materials characterization. RHEED was used to monitor the surface reconstruction of the $\varepsilon$-Ge layer during the oxide desorption, as well as the upper $\mathrm{In}_{x} \mathrm{Ga}_{1-x}$ As layer growth. The thorough desorption of native oxides from the $\varepsilon$-Ge epilayer is crucial in achieving an atomically abrupt, oxide-free heterointerface, thereby reducing the likelihood of generating electrically or optically active interfacial defect states.

Materials Characterization. To determine the crystalline quality and relaxation state of the tensile-strained epitaxial Ge layers grown on $\mathrm{Si}$, high-resolution triple axis X-ray rocking curves and reciprocal space maps were recorded from each sample using a Panalytical X-pert Pro system equipped with both PIXel and proportional detectors and a monochromated $\mathrm{Cu} \mathrm{K \alpha}(\lambda=1.540598 \AA) \mathrm{X}$-ray source. Select samples underwent further $\mathrm{X}$-ray diffraction characterization following removal of the $\varepsilon$-Ge epilayer using an $\mathrm{NH}_{4} \mathrm{OH}: \mathrm{H}_{2} \mathrm{O}_{2}: \mathrm{H}_{2} \mathrm{O}(2: 1: 200$ volume ratio) wet etch. Raman spectra acquired using a JY Horiba LabRam HR800 system equipped with a $514.32 \mathrm{~nm}$ Ar laser excitation source were used to independently confirm the strain-state of the $\varepsilon$-Ge thin films. Cross-sectional high-resolution transmission electron microscopy (HR-TEM) was used to characterize the long-range structural quality of each sample as well as the coherence and abruptness of each $\varepsilon$-Ge $/ \operatorname{In}_{x} \mathrm{Ga}_{1-x}$ As heterointerface. HR-TEM imaging was performed utilizing a JEOL 2100 transmission electron microscope. For this purpose, electron transparent foils of thin film cross sections of each sample structure were prepared by standard polishing techniques, i.e. mechanical grinding, dimpling and lowtemperature $\mathrm{Ar}^{+}$ion beam milling. To analyze the elemental composition of the structure, energy dispersive X-ray spectroscopy (EDS) was performed using a JEOL 2100 system operating in scanning TEM mode. The bandgap as a function of strain within each $\varepsilon$-Ge epilayer was characterized via microphotoluminescence spectroscopy using an $800 \mathrm{~nm}$ laser source and a thermoelectric cooled InGaAs detector for optical pumping and detection, respectively. The band alignment at each $\varepsilon$-Ge/ $/ n_{x} \mathrm{Ga}_{1-x}$ As heterointerface was investigated using a PHI Quantera SXM XPS system with a monochromatic $\mathrm{Al}-\mathrm{K} \alpha(E=1486.7 \mathrm{eV}) \mathrm{X}$-ray source. Samples with the upper $\operatorname{In}_{x} \mathrm{Ga}_{1-x}$ As layer growth were decapped using an $\mathrm{NH}_{4} \mathrm{OH}: \mathrm{H}_{2} \mathrm{O}_{2}: \mathrm{H}_{2} \mathrm{O}$ (2:1:200 volume ratio) wet etch prior to loading in the XPS chamber. The queue time was minimized between the cleaning of each $\varepsilon$-Ge layer or removal of the $\operatorname{In}_{x} \mathrm{Ga}_{1-x}$ As capping layer and loading into the XPS chamber. Each $\varepsilon$-Ge layer was thinned down to $1.5-2 \mathrm{~nm}$ for XPS measurements requiring photoelectron collection from the $\varepsilon$-Ge $/ \operatorname{In}_{x} \mathrm{Ga}_{1-x}$ As heterointerface. The $\mathrm{Ge} 3 \mathrm{~d}$ and As $3 \mathrm{~d}_{5 / 2}$ CL binding energy spectra, as well as $\varepsilon$-Ge and $\mathrm{In}_{x} \mathrm{Ga}_{1-x}$ As valence band binding energy spectra were collected with a pass energy of $26 \mathrm{eV}$ and an exit angle of $45^{\circ}$. Curve fitting was performed by CasaXPS v2.3.14 using a Lorentzian convolution with a Shirley-type background. The CL energy position was defined to be the center of the peak width at half of the peak height. The VBM values were determined by linear extrapolation of the leading edge to the baseline of the valence band spectra recorded for the $\varepsilon$-Ge and $\mathrm{In}_{x} \mathrm{Ga}_{1-x}$ As layers. The VBM value is sensitive to the choice of points on the leading edge used to obtain the regression line, resulting in an uncertainty in the range of $0.05-0.1 \mathrm{eV}$ for $\Delta E_{\mathrm{v}}$ and $\Delta E_{\mathcal{c}}$ respectively, as determined by regressions analysis of selected data over the linear region.

\section{AUTHOR INFORMATION}

\section{Corresponding Author}

*Tel: (540) 231-6663. Fax: (540) 231-3362. E-mail: mantu. hudait@vt.edu.

\section{Notes}

The authors declare no competing financial interest.

\section{ACKNOWLEDGMENTS}

M.C. acknowledges financial support from the NSF under grant number ECCS-1348653. P.G. acknowledges support from an NSF Graduate Research Fellowship Grant No. DGE. 0822220. The authors also acknowledge Dr. Luke F. Lester for several insightful technical discussions related to this work. Lastly, the authors acknowledge the NCFL-Institute for Critical Technology and Applied Science, Virginia Tech Nanofabrication facilities, and Charles Farley for assistance in materials characterization.

\section{REFERENCES}

(1) International Technology Roadmap for Semiconductors, 2013. h t t p : / / w w w.itrs.net/ I T R S \% 201999 2014\%20Mtgs,\%20Presentations\%20\&\%20Links/2013ITRS/ Summary2013.htm.

(2) Chau, R.; Doyle, B.; Datta, S.; Kavalieros, J.; Zhang, K. Integrated Nanoelectronics for the Future. Nat. Mater. 2007, 6, 810-812.

(3) Heyns, M.; Alian, A.; Brammertz, G.; Caymax, M.; Chang, Y. C.; Chu, L. K.; De Jaeger, B.; Eneman, G.; Gencarelli, F.; Groeseneken, G.; Hellings, G.; Hikavyy, A.; Hoffmann, T. Y.; Houssa, M.; Huyghebaert, C.; Leonelli, D.; Lin, D.; Loo, R.; Magnus, W.; Merckling, C.; Meuris, M.; Mitard, J.; Nyns, L.; Orzali, T.; Rooyackers, R.; Sioncke, S.; Soree, B.; Sun, X.; Vandooren, A.; Verhulst, A. S.; Vincent, B.; Waldron, N.; Wang, G.; Wang, W. E.; Witters, L. Advancing CMOS Beyond the Si Roadmap with Ge and 
III/V Devices. IEEE Int. Electron Devices Meet. (IEDM) 2011, DOI: 10.1109/IEDM.2011.6131543.

(4) Chau, R.; Datta, S.; Majumdar, A. Opportunities and Challenges of III-V Nanoelectronics for Future High-Speed, Low-Power Logic Applications. IEEE Compd. Semicond. Integr. Circuit Symp., IEEE CSICS, Tech. Dig. 2005, DOI: 10.1109/CSICS.2005.1531740.

(5) Zhang, Q.; Fang, T.; Xing, H.; Seabaugh, A.; Jena, D. Graphene Nanoribbon Tunnel Transistors. IEEE Electron Device Lett. 2008, 29, 1344-1346.

(6) Kavalieros, J.; Doyle, B.; Datta, S.; Dewey, G.; Doczy, M.; Jin, B.; Lionberger, D.; Metz, M.; Rachmady, W.; Radosavljevic, M.; Shah, U.; Zelick, N.; Chau, R. Tri-Gate Transistor Architecture with High-k Gate Dielectrics, Metal Gates and Strain Engineering. Int. Symp. VLSI Technol., Syst., Appl., Tech. Dig. 2006, DOI: 10.1109/ VLSIT.2006.1705211.

(7) Seabaugh, A. C.; Zhang, Q. Low-Voltage Tunnel Transistors for Beyond CMOS Logic. Proc. IEEE 2010, 98, 2095-2110.

(8) Ionescu, A. M.; Riel, H. Tunnel Field-Effect Transistors as Energy-efficient Electronic Switches. Nature 2011, 479, 329-337.

(9) Auth, C. P.; Plummer, J. D. Scaling Theory for Cylindrical, FullyDepleted, Surrounding-Gate MOSFET's. IEEE Electron Device Lett. 1997, 18, 74-76.

(10) Datta, S.; Dewey, G.; Fastenau, J. M.; Hudait, M. K.; Loubychev, D.; Liu, W. K.; Radosavljevic, M.; Rachmady, W.; Chau, R. UltrahighSpeed 0.5 V Supply Voltage $\operatorname{In}_{0.7} \mathrm{Ga}_{0.3}$ As Quantum-Well Transistors on Silicon Substrate. IEEE Electron Device Lett. 2007, 28, 685-687.

(11) Yang, J. J.; Pickett, M. D.; Li, X.; Ohlberg, D. A. A.; Stewart, D. R.; Williams, R. S. Memristive Switching Mechanism for Metal/Oxide/ Metal Nanodevices. Nat. Nanotechnol. 2008, 3, 429-433.

(12) Miller, D. A. B. Device Requirements for Optical Interconnects to Silicon Chips. Proc. IEEE 2009, 97, 1166-1185.

(13) Miller, D. A. B. Physical Reasons for Optical Interconnection. Int. J. Optoelectronics 1997, 11, 155-168.

(14) Liang, D.; Bowers, J. E. Recent Progress in Lasers on Silicon. Nat. Photonics 2010, 4, 511-517.

(15) Yang, J.; Bhattacharya, P.; Wu, Z. Monolithic Integration of InGaAs-GaAs Quantum-Dot Laser and Quantum-Well Electroabsorption Modulator on Silicon. IEEE Photonics Technol. Lett. 2007, 19, 747-749.

(16) Jongthammanurak, S.; Liu, J.; Wada, K.; Cannon, D. D.; Danielson, D. T.; Pan, D.; Kimerling, L. C.; Michel, J. Large ElectroOptic Effect in Tensile Strained Ge-on-Si Films. Appl. Phys. Lett. 2006, 89, 161115.

(17) Kuo, Y.-H.; Lee, Y. K.; Ge, Y.; Ren, S.; Roth, J. E.; Kamins, T. I.; Miller, D. A. B.; Harris, J. S. Strong Quantum-Confined Stark Effect in Germanium Quantum-Well Structures on Silicon. Nature 2005, 437, 1334-1336.

(18) Ahn, D.; Hong, C.-Y.; Liu, J.; Giziewicz, W.; Beals, M.; Kimerling, L. C.; Michel, J.; Chen, J.; Kartner, F. X. High Performance, Waveguide Integrated Ge Photodetectors. Opt. Express 2007, 15, 3916-3921.

(19) Vivien, L.; Rouviere, M.; Fedeli, J.-M.; Marris-Morini, D.; Damlencourt, J.-F.; Mangeney, J.; Crozat, P.; El Melhaoui, L.; Cassan, E.; Le Roux, X.; Pascal, D.; Laval, S. High Speed and High Responsivity Germanium Photodetector Integrated in a Silicon-OnInsulator Microwaveguide. Opt. Express 2007, 15, 9843-9848.

(20) Roth, J. E.; Fidaner, O.; Schaevitz, R. K.; Kuo, Y.-H.; Kamins, T. I.; Harris, J. S.; Miller, D. A. B. Optical Modulator on Silicon Employing Germanium Quantum Wells. Opt. Express 2007, 15, 58515859.

(21) Feng, N.-N.; Feng, D.; Liao, S.; Wang, X.; Dong, P.; Liang, H.; Kung, C.-C.; Qian, W.; Fong, J.; Shafiiha, R.; Luo, Y.; Cunningham, J.; Krishnamoorthy, A. V.; Asghari, M. $30 \mathrm{GHz}$ Ge Electro-Absorption Modulator Integrated with $3 \mu \mathrm{m}$ Silicon-on-Insulator Waveguide. Opt. Express 2011, 19, 7062-7067.

(22) Liu, J.; Sun, X.; Camacho-Aguilera, R.; Kimerling, L. C.; Michel, J. Ge-on-Si Laser Operating at Room Temperature. Opt. Lett. 2010, $35,679-681$.
(23) Camacho-Aguilera, R. E.; Cai, Y.; Patel, N.; Bessette, J. T.; Romagnoli, M.; Kimerling, L. C.; Michel, J. An Electrically Pumped Germanium Laser. Opt. Express 2012, 20, 11316-11320.

(24) Cho, S.; Park, B.-G.; Yang, C.; Cheung, S.; Yoon, E.; Kamins, T. I.; Yoo, S. J. B.; Harris, J. S. Room-Temperature Electroluminescence from Germanium in an $\mathrm{Al}_{0.3} \mathrm{Ga}_{0.7} \mathrm{As} / \mathrm{Ge}$ Heterojunction LightEmitting Diode by $\Gamma$-Valley Transport. Opt. Express 2012, 20, 14921-14927.

(25) Assefa, S.; Xia, F.; Vlasov, Y. A. Reinventing Germanium Avalanche Photodetector for Nanophotonic On-Chip Optical Interconnects. Nature 2010, 464, 80-84.

(26) Mi, Z.; Yang, J.; Bhattacharya, P.; Qin, G.; Ma, Z. HighPerformance Quantum Dot Lasers and Integrated Optoelectronics on Si. Proc. IEEE 2009, 97, 1239-1249.

(27) Fedeli, J.-M.; Bakir, B. B.; Olivier, N.; Grosse, Ph.; Grenouillet, L.; Augendre, E.; Philippe, P.; Bordel, K. D.; Harduin, J. InP on SOI Devices for Optical Communication and Optical Network on Chip. Proc. SPIE 2011, 7942, 794200.

(28) Duan, G.-H.; Jany, C.; Le Liepvre, A.; Accard, A.; Lamponi, M.; Make, D.; Kaspar, P.; Levaufre, G.; Girard, N.; Lelarge, F.; Fedeli, J.M.; Messaoudene, S.; Bordel, D.; Olivier, S. Hybrid III-V on Silicon Lasers for Photonic Integrated Circuits on Silicon. Proc. SPIE 2014, 9002, 90020X.

(29) Wirths, S.; Geiger, R.; von den Driesch, N.; Mussler, G.; Stoica, T.; Mantl, S.; Ikonic, Z.; Luysberg, M.; Chiussi, S.; Hartmann, J. M.; Sigg, H.; Faist, J.; Buca, D.; Grutzmacher, D. Lasing in Direct-Bandgap GeSn Alloy Grown on Si. Nat. Photonics 2015, 9, 88-92.

(30) Wirths, S.; Tiedemann, A. T.; Ikonic, Z.; Harrison, P.; Hollander, B.; Stoica, T.; Mussler, G.; Hartmann, M.; Grutzmacher, D.; Buca, D.; Mantl, S. Band Engineering and Growth of Tensile Strained $\mathrm{Ge} /(\mathrm{Si}) \mathrm{GeSn}$ Heterostructure Tunnel Field Effect Transistors. Appl. Phys. Lett. 2013, 102, 192103.

(31) Wirths, S.; Stange, D.; Pampillon, M.-A.; Tiedemann, A. T.; Mussler, G.; Fox, A.; Breuer, U.; Baert, B.; San Andres, E.; Nguyen, N. D.; Hartmann, J.-M.; Ikonic, Z.; Mantl, S.; Buca, D. High-k Gate Stacks on Low Bandgap Tensile Strained Ge and GeSn Alloys for Field-Effect Transistors. ACS Appl. Mater. Interfaces 2015, 7, 62-67.

(32) Madelung, O. Semiconductors: Intrinsic Properties of Group IV Elements and III-V, II-VI, and I-VII Compounds, Vol. 22a; Springer: Berlin, 1985.

(33) Hudait, M. K.; Lin, Y.; Ringel, S. A. Strain Relaxation Properties of InAs $\mathrm{P}_{1-\mathrm{y}}$ Metamorphic Materials Grown on InP Substrates. J. Appl. Phys. 2009, 105, 061643.

(34) Ghani, T.; Armstrong, M.; Auth, C.; Bost, M.; Charvat, P.; Glass, G.; Hoffmann, T.; Johnson, K.; Kenyon, C.; Klaus, J.; McIntyre, B.; Mistry, K.; Murthy, A.; Sandford, J.; Silberstein, M.; Sivakumar, S.; Smith, P.; Zawadzki, K.; Thompson, S.; Bohr, M. A 90nm High Vol. Manufacturing Logic Technology Featuring Novel 45nm Gate Length Strained Silicon CMOS Transistors. IEEE Int. Electron Devices Meet. (IEDM) 2003, DOI: 10.1109/IEDM.2003.1269442.

(35) Ge, C.-H.; Lin, C.-C.; Ko, C.-H.; Huang, C.-C.; Huang, Y.-C.; Chan, B.-W.; Perng, B.-C.; Sheu, C.-C.; Tsai, P.-Y.; Yao, L.-G.; Wu, C.L.; Lee, T.-L.; Chen, C.-J.; Wang, C.-T.; Lin, S.-C.; Yeo, Y.-C.; Hu, C. Process-Strained Si (PSS) CMOS Technology Featuring 3D Strain Engineering. IEEE Int. Electron Devices Meet. (IEDM) 2003, DOI: 10.1109/IEDM.2003.1269442.

(36) Sanchez-Perez, J. R.; Boztug, C.; Chen, F.; Sudradjat, F. F.; Paskiewicz, D. M.; Jacobson, R. B.; Lagally, M. G.; Paiella, R. DirectBandgap Light-Emitting Germanium in Tensilely Strained Nanomembranes. Proc. Natl. Acad. Sci. U. S. A. 2011, 108, 18893-18898.

(37) Kash, K.; Worlock, J. M.; Sturge, M. D.; Grabbe, P.; Harbison, J. P.; Scherer, A.; Lin, P. S. D. Strain-Induced Lateral Confinement of Excitons in GaAs-AlGaAs Quantum Well Microstructures. Appl. Phys. Lett. 1988, 53, 782-784.

(38) Ouyang, Q.; Yang, M.; Holt, J.; Panda, S.; Chen, H.; Utomo, H.; Fischetti, M.; Rovedo, N.; Li, J.; Klymko, N.; Wildman, H.; Kanarsky, T.; Costrini, G.; Fried, D. M.; Bryant, A.; Ott, J. A.; Ieong, M.; Sung, C.-Y. Investigation of CMOS Devices with Embedded SiGe Source/ 
Drain on Hybrid Orientation Substrates. Int. Symp. VLSI Technol., Syst., Appl., Tech. Dig. 2005, DOI: 10.1109/.2005.1469199.

(39) Bai, Y.; Lee, K. E.; Cheng, C.; Lee, M. L.; Fitzgerald, E. A. Growth of Highly Tensile-Strained Ge on Relaxed $\mathrm{In}_{\mathrm{x}} \mathrm{Ga}_{1-\mathrm{x}} \mathrm{As}$ by Metal-Organic Chemical Vapor Deposition. J. Appl. Phys. 2008, 104, 084518.

(40) Fang, Y.-Y.; Tolle, J.; Roucka, R.; Chizmeshya, A. V. G.; Kouvetakis, J.; D’Costa, V. R.; Menendez, J. Perfectly Tetragonal, Tensile-Strained $\mathrm{Ge}$ on $\mathrm{Ge}_{1-\mathrm{y}} \mathrm{Sn}_{\mathrm{y}}$ Buffered $\mathrm{Si}(100)$. Appl. Phys. Lett. 2007, 90, 061915.

(41) Nguyen, P. D.; Clavel, M.; Goley, P. S.; Liu, J.-S.; Allen, N. P.; Guido, L. J.; Hudait, M. K. Heteroepitaxial Ge MOS Devices on $\mathrm{Si}$ Using Composite AlAs/GaAs Buffer. IEEE J. Electron Devices Soc. 2015, 3, 341-348.

(42) El Kurdi, M.; Bertin, H.; Martincic, E.; de Kersauson, M.; Fishman, G.; Sauvage, S.; Bosseboeuf, A.; Boucaud, P. Control of Direct Band Gap Emission of Bulk Germanium by Mechanical Tensile Strain. Appl. Phys. Lett. 2010, 96, 041909.

(43) Cheng, T.-H.; Ko, C.-Y.; Chen, C.-Y.; Peng, K.-L.; Luo, G.-L.; Liu, C. W.; Tseng, H.-H. Competitiveness Between Direct and Indirect Radiative Transitions of Ge. Appl. Phys. Lett. 2010, 96, 091105.

(44) Grzybowski, G.; Roucka, R.; Mathews, J.; Jiang, L.; Beeler, R. T.; Kouvetakis, J.; Menendez, J. Direct Versus Indirect Optical Recombination in Ge Films Grown on Si Substrates. Phys. Rev. B: Condens. Matter Mater. Phys. 2011, 84, 205307.

(45) Schmidt, T.; Lischka, K.; Zulehner, W. Excitation-Power Dependence of the Near-Band-Edge Photoluminescence of Semiconductors. Phys. Rev. B: Condens. Matter Mater. Phys. 1992, 45, 89898994.

(46) van Driel, H. M.; Elci, A.; Bessey, J. S.; Scully, M. O. Photoluminescence Spectra of Germanium at High Excitation Intensities. Solid State Commun. 1976, 20, 837-840.

(47) Klingenstein, W.; Schweizer, H. Direct Gap Recombination in Germanium at High Excitation Level and Low Temperature. SolidState Electron. 1978, 21, 1371-1374.

(48) Manna, S.; Katiyar, A.; Aluguri, R.; Ray, S. K. Temperature Dependent Photoluminescence and Electroluminescence Characteristics of Core-Shell Ge-GeO2 Nanowires. J. Phys. D: Appl. Phys. 2015, $48,215103$.

(49) Huo, Y.; Lin, H.; Chen, R.; Makarova, M.; Rong, Y.; Li, M.; Kamins, T. I.; Vuckovic, J.; Harris, J. S. Strong Enhancement of Direct Transition Photoluminescence with Highly Tensile-Strained Ge Grown by Molecular Beam Epitaxy. Appl. Phys. Lett. 2011, 98, 011111.

(50) Virgilio, M.; Manganelli, C. L.; Grosso, G.; Schroeder, T.; Capellini, G. Photoluminescence, Recombination Rate, and Gain Spectra in Optically Excited n-type and Tensile Strained Germanium Layers. J. Appl. Phys. 2013, 114, 243102.

(51) Cai, Y.; Han, Z.; Wang, X.; Camacho-Aguilera, R. E.; Kimerling, L. C.; Michel, J.; Liu, J. Analysis of Threshold Current Behavior for Bulk and Quantum-Well Germanium Laser Structures. IEEE J. Sel. Top. Quantum Electron. 2013, 19, 1901009.

(52) Pizzi, G.; Virgilio, M.; Grosso, G. Tight-Binding Calculation of Optical Gain in Tensile Strained [001]-Ge/SiGe Quantum Wells. Nanotechnology 2010, 21, 055202.

(53) Chang, S.-W.; Chuang, S. L. Theory of Optical Gain of Ge$\mathrm{Si}_{\mathrm{x}} \mathrm{Ge}_{\mathrm{y}} \mathrm{Sn}_{1-\mathrm{x}-\mathrm{y}}$ Quantum-Well Lasers. IEEE J. Quantum Electron. 2007, $43,249-256$.

(54) Sorianello, V.; Perna, A.; Colace, L.; Assanto, G.; Luan, H. C.; Kimerling, L. C. Near-Infrared Absorption of Germanium Thin Films on Silicon. Appl. Phys. Lett. 2008, 93, 111115.

(55) Liu, J.; Sun, X.; Kimerling, L. C.; Michel, J. Direct-gap Optical Gain of Ge on Si at Room Temperature. Opt. Lett. 2009, 34, 17381740.

(56) Lieten, R. R.; Bustillo, K.; Smets, T.; Simoen, E.; Ager, J. W.; Haller, E. E.; Locquet, J.-P. Photoluminescence of Bulk Germanium. Phys. Rev. B: Condens. Matter Mater. Phys. 2012, 86, 035204.
(57) Thomas, G. A.; Blount, E. I.; Capizzi, M. Indirect Recombination Mechanisms in Germanium. Phys. Rev. B: Condens. Matter Mater. Phys. 1979, 19, 702-718.

(58) Nishino, T.; Takeda, M.; Hamakawa, Y. Indirect Exciton Absorption in Germanium. J. Phys. Soc. Jpn. 1974, 37, 1016-1023.

(59) Pavarelli, N.; Ochalski, T. J.; Murphy-Armando, F.; Huo, Y.; Schmidt, M.; Huyet, G.; Harris, J. S. Optical Emission of a Strained Direct-Band-Gap Ge Quantum Well Embedded Inside InGaAs Alloy Layers. Phys. Rev. Lett. 2013, 110, 177404.

(60) Jin, S.; Zheng, Y.; Li, A. Characterization of Photoluminescence Intensity and Efficiency of Free Excitons in Semiconductor Quantum Well Structures. J. Appl. Phys. 1997, 82, 3870-3873.

(61) El Kurdi, M.; Fishman, G.; Sauvage, S.; Boucaud, P. Band Structure and Optical Gain of Tensile-Strained Germanium Based on a 30 Band k.p Formalism. J. Appl. Phys. 2010, 107, 013710.

(62) Kraut, E. A.; Grant, R. W.; Waldrop, J. R.; Kowalczyk, S. P. Precise Determination of the Valence-Band Edge in X-Ray Photoemission Spectra: Application to Measurement of Semiconductor Interface Potentials. Phys. Rev. Lett. 1980, 44, 1620-1623.

(63) Clavel, M.; Goley, P. S.; Jain, N.; Zhu, Y.; Hudait, M. K. Strain Engineered Biaxial Tensile Epitaxial Germanium for High-Performance Ge/InGaAs Tunnel Field-Effect Transistors. IEEE J. Electron Devices Soc. 2015, 3, 184-193.

(64) Zhu, Y.; Maurya, D.; Priya, S.; Hudait, M. K. Tensile-Strained Nanoscale $\mathrm{Ge} / \mathrm{In}_{0.16} \mathrm{Ga}_{0.84}$ As Heterostructure for Tunnel Field-Effect Transistor. ACS Appl. Mater. Interfaces 2014, 6, 4947-4953.

(65) Liu, J.-S.; Zhu, Y.; Goley, P. S.; Hudait, M. K. Heterointerface Engineering of Broken-Gap InAs/GaSb Multilayer Structures. ACS Appl. Mater. Interfaces 2015, 7, 2512-2517.

(66) Hudait, M. K.; Clavel, M.; Goley, P. S.; Jain, N.; Zhu, Y. Heterogeneous Integration of Epitaxial Ge on Si Using AlAs/GaAs Buffer Architecture: Suitability for Low-Power Fin Field-Effect Transistors. Sci. Rep. 2014, 4, 6964.

(67) Hudait, M. K.; Clavel, M.; Zhu, Y.; Goley, P. S.; Kundu, S.; Maurya, D.; Priya, S. Integration of $\mathrm{SrTiO}_{3}$ on Crystallographically Oriented Epitaxial Germanium for Low-Power Device Applications. ACS Appl. Mater. Interfaces 2015, 7, 5471-5479.

(68) Zhu, Y.; Clavel, M.; Goley, P. S.; Hudait, M. K. Growth, Strain Relaxation Properties and High-k Dielectric Integration of MixedAnion GaAs $s_{1-y} \mathrm{Sb}_{\mathrm{y}}$ Metamorphic Materials. J. Appl. Phys. 2014, 116, 134304.

(69) Paul, S.; Roy, J. B.; Basu, P. K. Empirical Expressions for the Alloy Composition and Temperature Dependence of the Band Gap and Intrinsic Carrier Density in $\mathrm{Ga}_{\mathrm{x}} \mathrm{In}_{1-\mathrm{x}}$ As. J. Appl. Phys. 1991, 69, $827-829$.

(70) Hudait, M. K.; Krupanidhi, S. B. Self-Annihilation of Antiphase Boundaries in GaAs Epilayers on Ge Substrates Grown by MetalOrganic Vapor-Phase Epitaxy. J. Appl. Phys. 2001, 89, 5972-5979. 